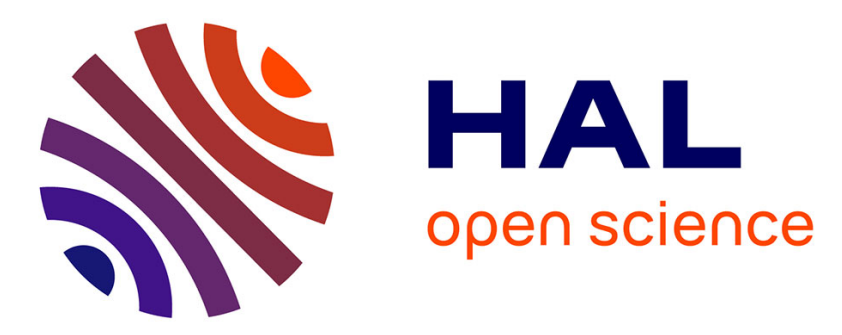

\title{
Fluorescence lifetime measurements applied to the characterization of the droplet temperature in sprays
}

Mehdi Stiti, Yangpeng Liu, Hadrien Chaynes, Fabrice Lemoine, Xishi Wang, Guillaume Castanet

\section{- To cite this version:}

Mehdi Stiti, Yangpeng Liu, Hadrien Chaynes, Fabrice Lemoine, Xishi Wang, et al.. Fluorescence lifetime measurements applied to the characterization of the droplet temperature in sprays. Experiments in Fluids, 2021, 62 (8), 10.1007/s00348-021-03264-x . hal-03410410

\section{HAL Id: hal-03410410 https://hal.science/hal-03410410}

Submitted on 3 Nov 2021

HAL is a multi-disciplinary open access archive for the deposit and dissemination of scientific research documents, whether they are published or not. The documents may come from teaching and research institutions in France or abroad, or from public or private research centers.
L'archive ouverte pluridisciplinaire HAL, est destinée au dépôt et à la diffusion de documents scientifiques de niveau recherche, publiés ou non, émanant des établissements d'enseignement et de recherche français ou étrangers, des laboratoires publics ou privés. 


\title{
Fluorescence lifetime measurements applied to the characterization of the droplet temperature in sprays
}

\author{
Stiti Mehdi ${ }^{1}$. Liu Yangpeng ${ }^{1,2}$. Chaynes Hadrien ${ }^{1} \cdot$ Lemoine Fabrice $^{1} \cdot$ Wang Xishi $^{2}$. \\ Castanet Guillaume ${ }^{1}$
}

\section{Introduction}

The characterization of the droplet temperature in polydisperse sprays is an important challenge in many engineering applications. The transient phenomena within droplets that are heating or cooling, and the heat and mass transfer to their surfaces have long been the subjects of intense research in liquid-fuelled combustion systems, spray cooling, spray drying, and many more processes related to sprays and liquid atomization in general. While the size and velocity of droplets can be measured using relatively widespread and well-established techniques, characterizing the temperature remains quite challenging (Lemoine and Castanet 2013). Several approaches relying upon a wide variety of physical principles have been brought in the recent decades and are still being actively pursued, like rainbow refractometry (Lemaitre et al. 2006; Saengkaew et al. 2018), Raman scattering (Carns et al. 1990; Schweiger 1990; Müller et al. 2000), IR thermography (Tuckermann et al. 2005; Brutin et al. 2011; Labergue et al. 2017), and laser-induced photoluminescence processes including laser-induced fluorescence (Lemoine and Castanet 2013) and laser-induced phosphorescence (Strizhak et al. 2020). Among these techniques, laserinduced fluorescence (LIF) offers unprecedent temperature sensitivities and a high flexibility for a wide range of applications. Examples include droplets spreading onto solid surfaces (Chaze et al. 2017), mono- and multi-component fuel droplets combusting and evaporating in high-temperature

Stiti Mehdi and Liu Yangpeng have contributed equally and should be considered co-first authors.

Castanet Guillaume

guillaume.castanet@univ-lorraine.fr

1 LEMTA, CNRS, Université de Lorraine, 54000 Nancy, France

2 State Key Lab. of Fire Science, University of Science and Technology of China, 230026 Hefei, People's Republic of China environments (Perrin et al. 2015), supercooled droplets in icing processes (Stiti et al. 2019). Usually, an organic dye is dissolved into the liquid prior to its injection. The droplets are illuminated by a laser beam with an appropriate wavelength to allow light absorption by the fluorescent molecules which emit in return a fluorescence signal depending on the temperature by some of its properties (intensity, wavelength, lifetime). A few dyes, such as rhodamine B or kiton red, have been used so far for temperature measurement in water solutions. Besides a high dependency of their fluorescence signal on temperature, these dyes have remarkably high absorption cross sections and high quantum fluorescence yields, which is advantageous for the implementation of the measurements.

LIF-based temperature measurements in both single and multiphase flows are usually based on the intensity of the fluorescence signal. However, intensity measurements cannot provide an absolute quantity. A reference, measured at a point where the temperature is already known, is always required. This reference must be carried out under experimental conditions close to the measurement conditions whenever it is possible. Any disturbance (in the intensity of the light source, in the background light, in the light transmission through the detection optics, or due to the dye photobleaching and degradation) will lead to some errors in the derived temperature. However, it is sometimes difficult to obtain a valid reference due to various constraints. For example, the liquid temperature can change significantly within some high-pressure spray nozzle in direct combustion engines due to the combined effects of high pressure and viscous frictions within the injector core (Wolff et al. 2007), leaving the injection temperature unknown.

Temperature measurements are particularly complex in multiphase flows like sprays. Indeed, the fluorescence intensity from a droplet depends on many parameters in addition to the liquid temperature such as the droplet size, the droplet position with respect to the laser beam and the detector, the concentration in dye for evaporating droplets (Frackowiak 
and Tropea 2010; Lemoine and Castanet 2013). As a result, it is generally unavoidable to use a ratiometric approach, i.e., to calculate the ratio of the intensities of the fluorescence signal collected in two spectral bands. Properly used, intensity ratios can cancel out most of the adverse effects encountered with droplets and thus provide the basis for accurate droplet temperature measurements in sprays. The so-called two-color LIF (2cLIF) technique has been implemented successfully as a pointwise method (Castanet et al. 2003, 2016; Perrin et al. 2015; Labergue et al. 2017; Stiti et al. 2019) and more recently as an imaging technique (Düwel et al. 2007; Chaze et al. 2016, 2017; Palmer et al. 2020). Whatever the chosen approach (pointwise or imaging), one of the issues is the selection of detection bands and fluorescent dyes. The 2 cLIF method can use either a single dye or two dyes in order to obtain two bands with different temperature sensitivities. The single dye approach takes advantage of the temperature deformation of the emission spectrum of dyes such as rhodamine B. However, this distortion is usually relatively small, and it is usually necessary to select very carefully two spectral bands having a sufficient good signal-noise ratio and different sensitivity to temperature. The two-dye approach requires two dyes that are sufficiently well separated in wavelength to avoid spectral conflicts, for example, the emission of one dye in the detection band of the other dye (Chaze et al. 2016). However, such a pair of dyes is not always straightforward to identify and energy transfer between the dye molecules (Förster 1948) can alter the dyes' emission and their response to temperature. In spray application of the 2cLIF technique, several sources of error have been reported (Labergue et al. 2010, 2012) due to the polydispersion in droplet size and multiple light scattering by the droplets. In particular, laser light scattering by the droplets can induce a significant out-of-field fluorescence which extends outside the focal plane of the collection optics and contributes to the overall fluorescence signal. The fluorescence signal attenuation can be amplified by the reabsorption of the fluorescence on certain bands. As an alternative, measuring the fluorescence lifetime provides a direct means to access to an intrinsic parameter that is related to the key mechanisms of the fluorescence cycle. The fluorescence lifetime is closely dependent on the quenching rate of the fluorescence and therefore on the temperature. Contrary the fluorescence intensity, the lifetime value is not dependent on the measurement method and system, whatever it is operating in the time domain by using a pulsed source or in the frequency domain by a phase-modulated light source. When a population of fluorescent molecules is excited by an ultrashort laser pulse, the time-resolved fluorescence signal decays exponentially with time. A standard method to determine the time rate of this decay is to use the so-called time-correlated single-photon counting (TCSPC) technique (Lakowicz 2013). The latter is basically a single-photon stopwatch at the nanosecond scale. Its implementation involves a high repetition rate pulsed laser, a single-photon detector, and sophisticated counting electronics. The applications of TCSPC are traditionally in the field of biology where it is performed on confocal or multiphoton laser scanning microscopes to reconstruct images of the fluorescence lifetime in living cells. The measurement process is relatively slow, as it usually takes several tens of seconds to acquire enough data to accurately determine the decay time. This poor temporal resolution can be a limiting factor for applications to fluid mechanics. Nonetheless, a few studies have recently dealt with moving droplets in microfluidic systems to investigate the mixing inside droplets immersed in a second immiscible liquid inside microchannels (Casadevall I Solvas et al. 2010; Zeng et al. 2011). In these studies, two fluorescence dyes are brought into contact during the formation process of the droplets inside a T-junction connecting several microchannels. As the two dyes have very different fluorescent lifetimes, their relative concentration can be determined from the consideration of a biexponential decay. Bennet et al. (Bennet et al. 2011) measured the fluorescence lifetime of kiton red to determine the temperature inside water droplets suspended by optical tweezers. To our best knowledge, nothing equivalent has been reported for the temperature of droplets freely moving in a gas medium, although measuring the fluorescence lifetime could offer many advantages. For instance, it would require a single dye and a single spectral detection band. The aim of the present study is to develop a very first application of TCSPC to measure the temperature of water droplets in a polydisperse spray. The spray selected for this application has already been used to carry out temperature measurements using the $2 \mathrm{cLIF}$ technique, and due to its characteristics in terms of size and density of the droplets, it has helped to highlight certain deficiencies and biases of the technique.

\section{The effect of temperature on the fluorescence lifetime}

Fluorescence results from the spontaneous emission of photons by molecules excited to an electronic state of higher energy than their ground state. In the case of laser-induced fluorescence, the excitation comes from the absorption of a laser radiation. In general, fluorescence emissions have a longer wavelength than the absorbed light. This Stokes shift is due to energy loss between the time a photon is absorbed and when it is emitted. Many pathways can compete with fluorescence including collisional quenching, intersystem crossing, internal conversion, quenching by energy transfer, and charge transfer reaction (Lakowicz 2013). All these processes contribute to a quenching of the fluorescence emission. The efficiency of photon emission through fluorescence 
is evaluated by the fluorescence quantum yield $\phi$ which is defined as the ratio of the number of photons emitted to the number of photons absorbed:

$\phi=\frac{k_{f}}{k_{f}+k_{\mathrm{nr}}}$

In this expression, $k_{f}\left[\mathrm{~s}^{-1}\right]$ denotes the rate of spontaneous emission. $k_{\mathrm{nr}}\left[\mathrm{s}^{-1}\right]$ is the rate of non-radiative decay by processes including the collisional quenching. Fluorescence typically follows a first-order kinetics. After an excitation by an ultrashort pulse of light, the fluorescence signal $I_{f}(t)$ emitted by the population of molecules will ideally decay with time according to:

$\frac{I_{f}(t)}{I_{f}(0)}=e^{-\frac{t}{\tau}}$,

where $\tau$ is the fluorescence lifetime, which is on the order a few nanoseconds for organic dyes that contain a xanthene core. However, there are quite a few instances in the literature where biexponential decays were used to describe the decay of some fluorophores including rhodamine B (Boens et al. 2007; Kristoffersen et al. 2014). In this case, the decay can be described using the following expression:

$\frac{I_{f}(t)}{I_{f}(0)}=a e^{-\frac{t}{\tau_{1}}}+(1-a) e^{-\frac{t}{\tau_{2}}}$,

where $a$ is a parameter between 0 and 1 . An average fluorescence lifetime $\tau$ for this type of decay can be computed as (Casadevall I Solvas et al. 2010):

$\tau=a \tau_{1}+(1-a) \tau_{2}$,

The rate of non-radiative pathways $k_{n r}$ modifies the excited state lifetime similarly as the fluorescence quantum yield $\phi$. The total decay rate $\tau$ is the sum over all the radiative and non-radiative decays rate:

$\tau=\left(k_{f}+k_{\mathrm{nr}}\right)^{-1}$,

The rate of spontaneous emission $k_{f}$ being a constant for an excitation wavelength, the lifetime $\tau$ is proportional to the fluorescence yield:

$\phi=\tau / \tau_{0}$,

where $\tau_{0}$ is the natural lifetime of the fluorophore without any quenching process. In the same way as the fluorescence quantum yield, the lifetime is sensitive to a large variety of physical properties including the temperature.

When the light excitation extends over a much longer period than the lifetime, the intensity of the signal fluorescence reaches a steady-state value that can be determined by:
$I_{f} \sim I_{0} \varepsilon_{0} N \phi$,

where $I_{0}\left[\mathrm{~W} \cdot \mathrm{m}^{-2}\right]$ is the fluence of the incident laser light which must stay well below the saturation threshold (Chaze et al. 2016). $N\left[\mathrm{~mol} . \mathrm{m}^{-3}\right]$ is the molar concentration of fluorescent molecules. $\varepsilon_{0}\left[\mathrm{~mol}^{-1} \cdot \mathrm{m}^{-2}\right]$ corresponds to the absorption coefficient at the laser wavelength. According to (Chaze et al. 2016), the temperature dependence of $I_{f}$ has two distinct origins, on the one hand the fluorescence quantum yield $\phi$ and on the other hand the variation in the absorption cross section $\varepsilon_{0}$. The quantum yield of fluorescence $\phi$ as well as the lifetime $\tau$ decreases with temperature because the nonradiative extinction rate $k_{n r}$ increases with the intermolecular collisions.

For some dyes excited at specific wavelengths, such as fluorescein illuminated at $532 \mathrm{~nm}$, the temperature variation in the absorption coefficient $\varepsilon_{0}$ can be predominant over the one of the fluorescence quantum yields $\phi$ (Chaze et al. 2016). However, this feature has no relevance when only the fluorescence lifetime is used for measuring the temperature. The dyes selected for this study are rhodamine $B(\mathrm{RhB})$ and kiton red (KR) (also named sulforhodamine B) because they have been extensively used for thermometry applications in water solutions using both intensity-based measurements and lifetime-based measurements with an accuracy of $0.5^{\circ} \mathrm{C}$ (Paviolo et al. 2013). The fluorescence quantum yield of a fluorophore is well known to depend on solvent effects through the non-radiative rate constant $k_{\mathrm{nr}}$. Effects of the solvent include a dynamic contribution caused by collisions with the solvent molecules and a static contribution arising from short-range chemical interactions. In the case of rhodamine $\mathrm{B}$, the temperature-dependent fluorescence quantum yield can be attributed to the flexibility of the diethylamino groups, which accelerates the rate of non-radiative decay by internal conversion (Casey and Quitevis 1988). Besides, an Arrhenius law has been widely accepted to describe the effect of temperature on the rate of the non-radiative decay for RhB and KR in many solvents (Mendels et al. 2008). For simplicity, this law was also considered for the temperature dependence of the fluorescence intensity and the lifetime (Mercadé-Prieto et al. 2017):

$$
\tau=A \exp \left(\frac{E_{a}}{\mathrm{RT}}\right),
$$

where $E_{a}$ is the activation energy, $A$ is a pre-exponential factor, and $R$ is the gas constant $\left(R=8.314 \mathrm{~J} \cdot \mathrm{K}^{-1} \cdot \mathrm{mol}^{-1}\right)$. For KR and RhB, values of $E_{a}$ have been reported by (Mercadé-Prieto et al. 2017) based on their own measurements and data taken from the literature. For RhB in water, $E_{a}$ was estimated at $21 \mathrm{~kJ} / \mathrm{mol}$ in the range $20-60{ }^{\circ} \mathrm{C}$ based on the data from Müller et al. (2009) and $15.4 \mathrm{~kJ} / \mathrm{mol}$ from Paviolo et al. (2013). For KR in water, Mendels et al. (2008) 
obtained a value of $E_{a}=24.2 \mathrm{~kJ} / \mathrm{mol}$. Given that $\phi \sim \tau$, the value of $E_{a}$ can be also derived from the intensity decrease in the signal with the temperature. Doing so, $E_{a}$ was evaluated in the range 18-22 kJ/mol (Smart and Laidlaw 1977; Ross et al. 2001; Natrajan and Christensen 2008). While the effect of the solvent and the chemical environment was examined in several studies (Zhang et al. 2014; Hilaire et al. 2017), little consideration has been given to the dependence of $E_{a}$ on the emission wavelength. However, this dependence is strong enough to make it possible to use $\mathrm{RhB}$ and $\mathrm{KR}$ to measure the temperature with the two-color one-dye method (Lemoine and Castanet 2013). Special care will be therefore placed on the evaluation of $E_{a}$ and $A$ (Eq. (8)) before applying the measurement technique to droplets.

\section{Fluorescence lifetime measurements in the time domain}

TCSPC is a widespread and effective method for measuring the fluorescence lifetime in the time domain with an ultrashort pulsed laser. The elapsed time between the sample excitation by an ultrashort pulsed light source and the emission of a fluorescence photon can be measured typically within a picosecond accuracy. Figure 1 shows the optical system used for the implementation of the TCSPC technique. The excitation of the fluorescent dyes is performed by a femtosecond Ti:Sapphire laser (Coherent Chameleon Ultra II) which is tunable from 690 to $1080 \mathrm{~nm}$ and generates light pulses with a width of about $140 \mathrm{fs}$. To induce the fluorescence of $\mathrm{RhB}$ and $\mathrm{KR}$, the laser is firstly tuned on a wavelength of $980 \mathrm{~nm}$. Then, frequency of the laser beam is doubled using the second harmonic generator HarmoniXX from A.P.E GmbH. The resulting wavelength centered around $490 \mathrm{~nm}$ allows obtaining an intense fluorescence signal with RhB and KR. When the measurements are performed in the spray, the laser beam is focused by means of an achromatic lens with a focal length $f=200 \mathrm{~mm}$, allowing a beam waist with a diameter of about $100 \mu \mathrm{m}$ and a Rayleigh length of about $6 \mathrm{~cm}$ to be obtained.

A fast hybrid photomultiplier tube (R10467, Hamamatsu) with a Peltier cooler to reduce the dark count rate is used for photon counting. The output of the detector is sent to a timecorrelating counter (HydraHarp 400 controller, PicoQuant $\mathrm{GmbH}$ ) which is operating in forward start stop mode to measure the arrival times of single photons. A laser pulse

(a) Lasing system (side view)

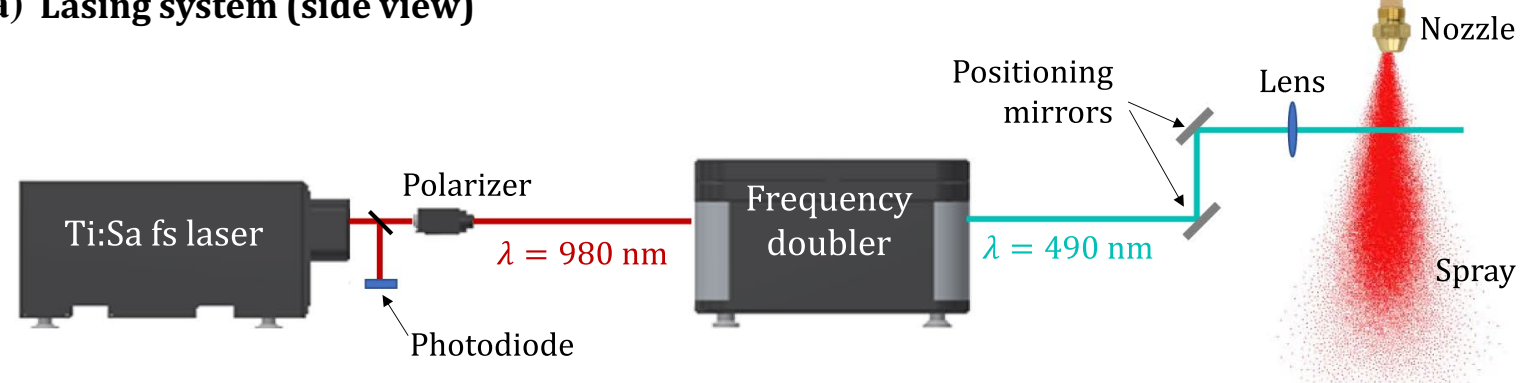

(b) Detection system (top view)

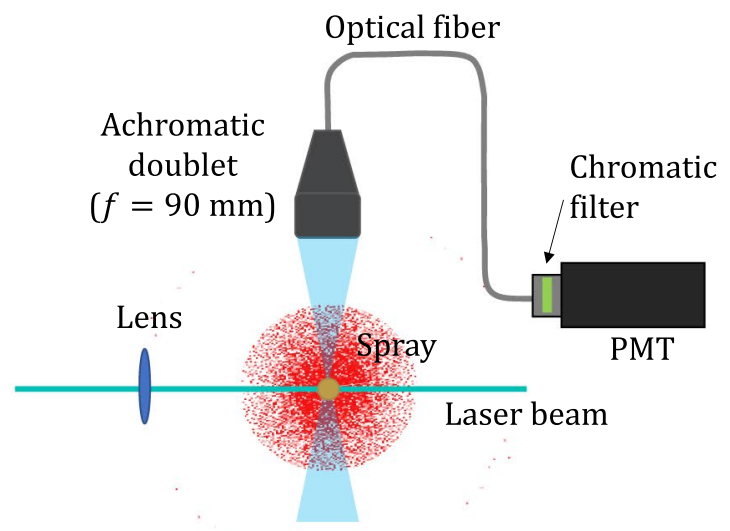

(c) Spraying system

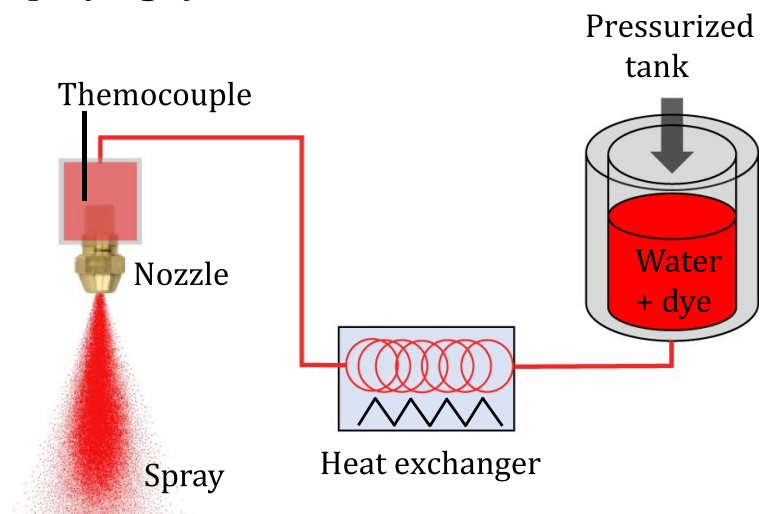

Fig. 1 Experimental setup 
causes a photon event at the detector. The electronics measures the delay between the laser pulse and the subsequent photon event. This measurement implies a sharp timing of the laser pulse, which is obtained from a fast photodiode (TDA 200 from PicoQuant $\mathrm{GmbH}$ ) connected to the timing electronics. This photodiode receives the reflected light from a beam splitter (99\% T, 1\% R) placed at the laser output and allows detecting the laser pulses with a small-time jitter due a very sharp falling edge (Fall time typ. $250 \mathrm{ps}$ ).

The fluorescence signal is collected at right angle from the laser excitation using an achromatic doublet with a focal length of $90 \mathrm{~mm}$, and the signal is transmitted by an optical fiber with a core diameter of $70 \mu \mathrm{m}$. The projected image of the core of the optical fiber is about $60 \mu \mathrm{m}$ width at the focal plane taking into account the optical magnification of the receiver front lens. The LIF measurement volume is defined as the intersection of the laser beam and the detection volume. It is roughly a $60-\mu \mathrm{m}$-long cylinder with a diameter of $100 \mu \mathrm{m}$. Finally, different bandpass filters can be mounted in front of the photomultiplier tube in order to detect the photons on specific spectral bands.

The basic principles of TCSPC have been discussed with great details in literature (Lakowicz 2013). The measurement method illustrated in Fig. 2 is based on the repetitive and precisely timed registration of single photons using the previously described system. By counting a high number of photon events to achieve statistical convergence, a histogram of the photon arrival times distribution can be built up. The width of the time bins in this histogram depends on the required time resolution (typically a few tens of ps) (Fig. 3).

A biexponential function was chosen for the fluorescence decay model to obtain a reliable value for the fluorescence decay $\tau$ regardless of the liquid temperature. Indeed, we found that the fluorescence decay is not always monoexponential and depended closely on the detection spectral band

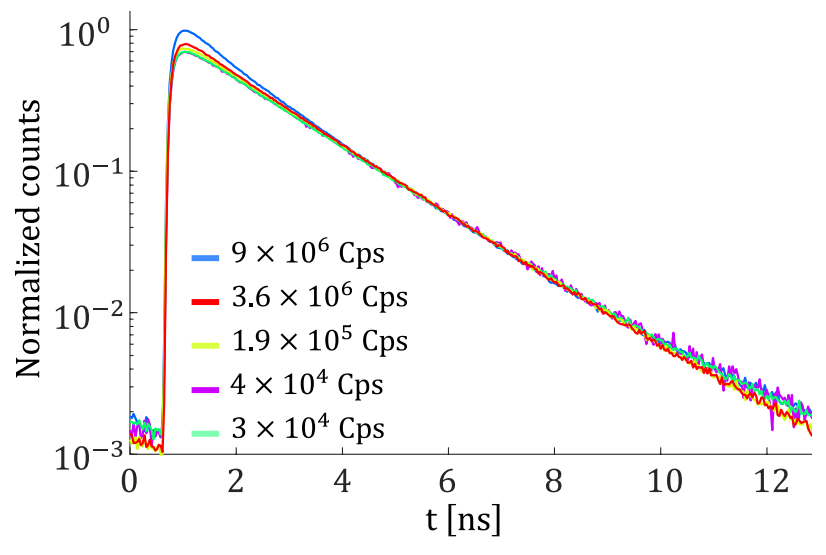

Fig. 3 Illustration of the pile-up effect. All the curves have been normalized to have the same value at $t=6 \mathrm{~ns}$. Due to the pile-up effect, the lifetime value is underestimated for the highest count rates. The results of the fitting are: $\tau=1.54 \mathrm{~ns}$ at $9 \mathrm{MCps}, \tau=1.71 \mathrm{~ns}$ at 3.6 MCps, $\tau=1.76 \mathrm{~ns}$ at $0.19 \mathrm{MCps}, \tau=1.77 \mathrm{~ns}$ at $40 \mathrm{kCps}$ and $\tau=$ $1.77 \mathrm{~ns}$ at $30 \mathrm{MCps}$, Measurements in a cell containing rhodamine B at $C=10^{-5} \mathrm{M}$ and $T=20^{\circ} \mathrm{C}$ in the spectral band [630 nm-650 nm]

as well as on the temperature as illustrated, for example, in Fig. 4. The method for determining the fluorescence lifetime is detailed in Appendix 1 where examples of fitting are presented. The goodness-of-the fit $\chi^{2}$ is evaluated by the least squares method. The instrument response function (IRF) of the TCSPC system is accounted for, as the decay model is first convoluted with the IRF before being compared to the experimental decay at any step of the fitting process.

A well-known limitation of TCSPC is that it is designed to record only one photon per excitation cycle. Detector and electronics have a dead time of several tens of nanoseconds after a photon event, meaning that photons arriving next during the dead time are completely lost. The dead time is typically longer than the fluorescence decay processes of (a)

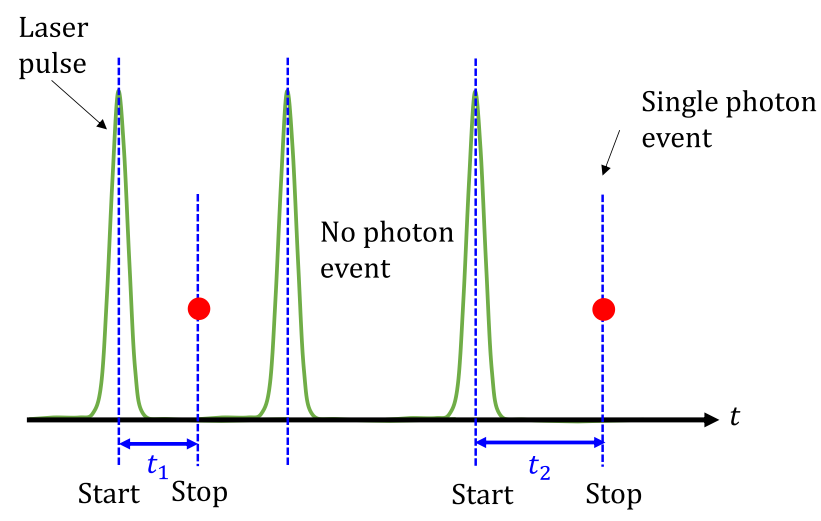

(b)

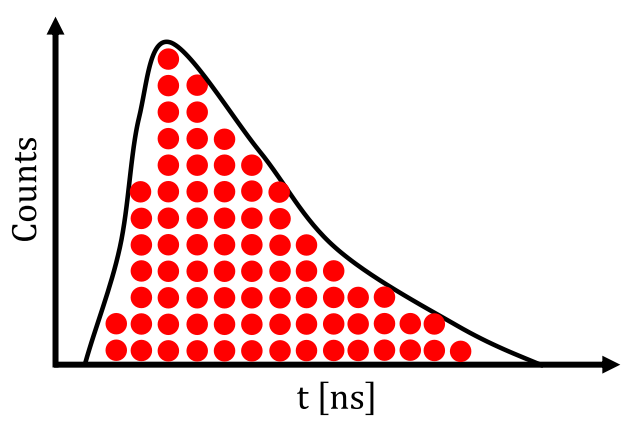

Fig. 2 Principle of the lifetime measurement with TCSPC. a TCSPC device typically measures the time between one START event and one STOP event (like a stopwatch). b A histogram of start-stop times is reconstructed by counting a high number of photon events to reach statistical convergence 

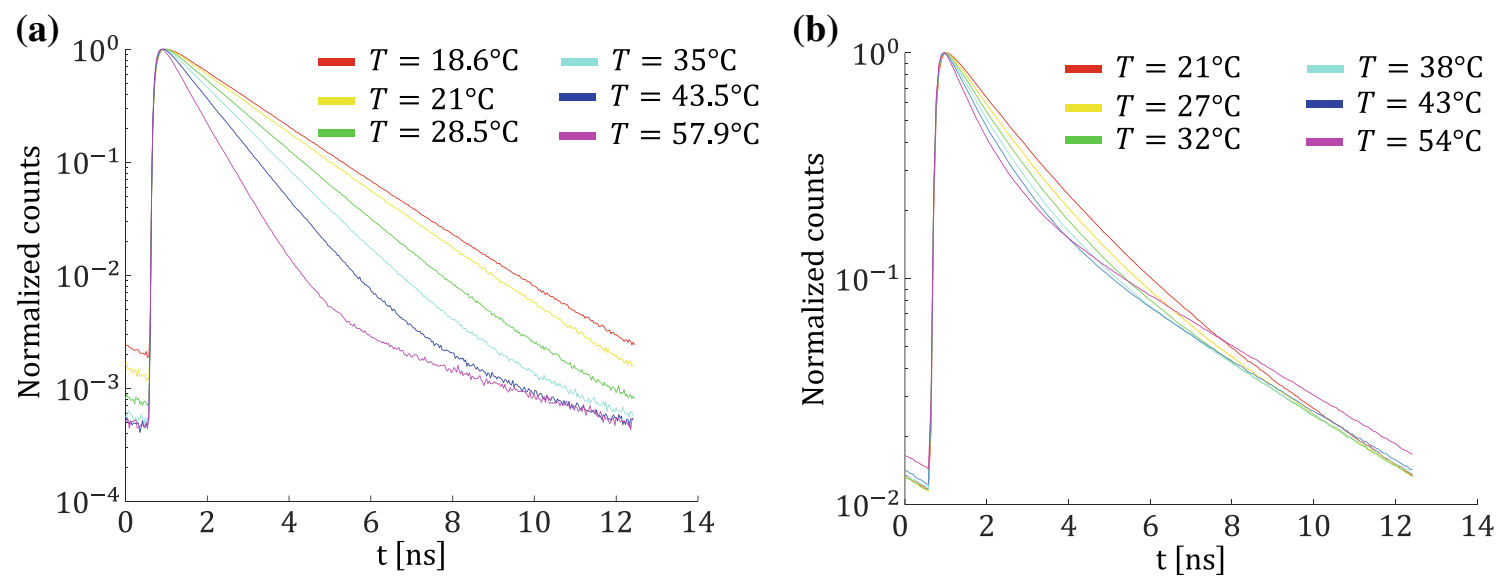

Fig. 4 Fluorescent decays of RhB at different temperatures for the spectral bands [630 nm - 650 nm] (a) and [540 nm - 560 nm] (b). Measurements carried out in a cell at a concentration of $10^{-5} \mathrm{M}$

interest. Consequently, if several photons are emitted per excitation cycle, only the first of them is detected. This causes a statistical over-representation of the earliest photons and leads to a distortion of the collected decay shape. This bias has been referred as pile-up effect (Becker 2005). In order to avoid this situation, it is necessary to work with sufficiently low laser light intensities. Due to the statistical nature of the emission of fluorescence photons, this results in the unfortunate situation that most of the excitation pulses lead to no emission at all and the rate of photon collection (count rate) is reduced drastically. In a cuvette, where the conditions are stationary, it was determined that the average detector count rate must not exceed $2.10^{6} \mathrm{Cps}$ (Count per second) with the present TCSPC system, while the laser has a $80 \mathrm{MHz}$ repetition rate. In sprays, the overall count rate cannot be a good indicator of the pile-up effect, whose occurrence is also dependent on the volume fraction of liquid (related to the droplet size crossing the probe volume) in the probe volume as well as light scattering by the droplets. In practice, to perform the measurements in sprays, the power of the laser is adjusted to have a count rate slightly below the pile-up limit near the spray nozzle outlet where the liquid fraction is the highest. Then, the laser power can be maintained for all the measurements downstream, even if the count rate is getting lower with the decrease in the spray density while the spray is expanding. The low dark count level combined with the wide dynamic range of the detector makes it possible to carry out measurements in spray regions where the volume fraction of the liquid reduces to a few thousandths in comparison with a liquid cuvette as it will be illustrated at the end of Sect. 4 .

\section{Temperature calibration}

Before measuring the droplet temperature in a spray, it is necessary to perform a calibration of the fluorescence lifetime as a function of temperature. This step consists in measuring the fluorescence lifetime in a cell for different liquid temperatures. The liquid is heated by means of a thermal resistance while it is continuously stirred to ensure a uniform temperature. A thermocouple is positioned a few $\mathrm{mm}$ from the LIF measurement volume. These calibration experiments were carried out mounting different optical filters in front of the PMT to set different spectral bands for fluorescence detection: [525 nm-535 nm], [540 nm-560 nm], $[570 \mathrm{~nm}-590 \mathrm{~nm}]$ and $[630 \mathrm{~nm}-650 \mathrm{~nm}]$. Figure 4 shows the fluorescent decay of KR at different temperatures for the spectral bands [540 nm-560 nm] and [630 nm-650 nm]. An important difference in temperature sensitivity can be observed. The fluorescence lifetime is clearly more sensible to the temperature in the red region of the fluorescence emission. In both cases, the fluorescence decay becomes more and more biexponential with the increase in the temperature.

Figure 5 shows the evolution of the lifetime in function of the temperature for different dyes and spectral bands tested in the calibrations. Rhodamine $6 \mathrm{G}(\mathrm{Rh} 6 \mathrm{G})$ has a longer lifetime than the other two dyes, but it is not sensitive to temperature, which was already observed with the signal intensity (Chaze et al. 2016). The quantum yield of Rh6G is known to be close to 0.95 in water, while that of $\mathrm{RhB}$ is about 0.3 at $20^{\circ} \mathrm{C}$ (Magde et al. 1999). On the average, the lifetime of Rh6G is longer than that of RhB, which seems consistent with its smaller quantum yield.

$\mathrm{RhB}$ and KR have quite similar behaviors. In the spectral band $[630 \mathrm{~nm}-650 \mathrm{~nm}]$, the two dyes have very close values of lifetime and comparable temperature sensitivities. In 
Fig. 5 Effect of the temperature on the fluorescence lifetimes of RhB, KR and rhodamine $6 \mathrm{G}$ (Rh6G) in different spectral bands of fluorescence emission. Measurements were carried out for dye concentrations of $10^{-5} \mathrm{M}$

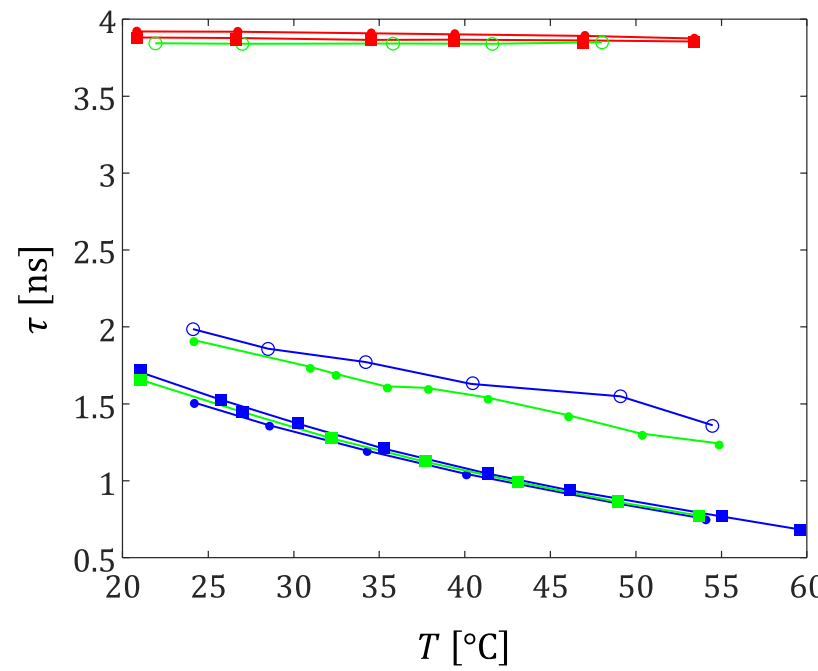

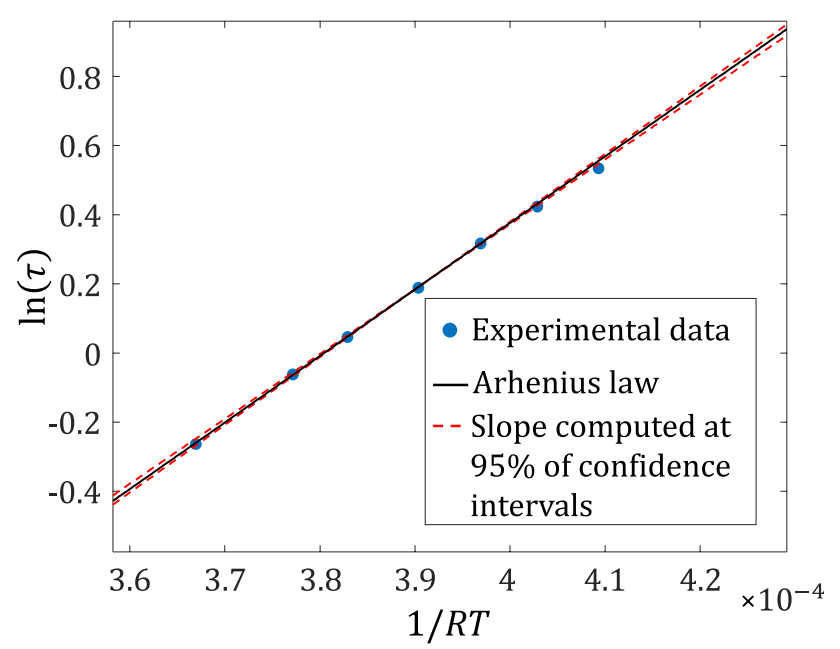

Fig. 6 Linear regression of $\ln (\tau)$ as a function of the inverse temperature to obtain the parameters of the Arrhenius equation $\left(E_{a}=18.7 \pm 0.4 \mathrm{~kJ} / \mathrm{mol}, A=6.69 \pm 0.85\right)$. Case of $\mathrm{KR}$ in the spectral band $[630 \mathrm{~nm}-650 \mathrm{~nm}]$ at a concentration of $10^{-6} \mathrm{M}$

the spectral band [540 nm-560 nm], the behavior of RhB remains unchanged, but KR is affected by the increase in its lifetime which remains quite sensitive to temperature. In the spectral band [525 nm-535 nm], KR loses its temperature sensitivity and it can be noticed that the lifetime takes a value very close to that of Rh6G. For RhB, the increase in lifetime becomes significant.

In Fig. $6, \ln (\tau)$ is plotted as a function of $1 / \mathrm{RT}$ for KR in the band $[630 \mathrm{~nm}-650 \mathrm{~nm}]$ and the linearity of the plot confirms the good suitability of the Arrhenus law (Eq. (8)). The temperature dependence of $\tau$ is controlled by the activation energy $E_{a}$ which can be determined from the slope of this curve.
It is also possible to determine the activation energy $E_{a}$ from intensity measurements. More details about these measurements are provided in Appendix 2. The deformation of the emission spectra of the fluorescence with the temperature reveals in the case of $\mathrm{RhB}$ and $\mathrm{KR}$ that the activation energy is indeed a function of the wavelength. In general, intensity and lifetime measurements give comparable results, although the values of $E_{a}$ obtained with the intensity measurements are slightly higher, especially in the spectral band $[570 \mathrm{~nm}-590 \mathrm{~nm}]$. These differences do not seem to be due to measurement errors. It could be attributed to different wavelengths of the lasers used to excite the dyes $(490 \mathrm{~nm}$ for the femtosecond laser, against $532 \mathrm{~nm}$ for the $\mathrm{CW}$ laser) and the wavelength dispersion of the femtosecond laser. However, according to Lakowicz et al. (2006), $E_{a}$ should be an intrinsic property of the dye in its solvent not depending on the initial perturbation conditions such as the wavelength of excitation, the duration of light exposure, the use of a multiphoton excitation, or the occurrence of photobleaching. The variation in $E_{a}$ with the wavelength was exploited in the two-color one-dye measurements method in which two emission bands of a single dye are used to calculate an intensity ratio $R$. Using Eqs. (6-8),

$R=\frac{I_{f_{1}}}{I_{f_{2}}}=\frac{\phi_{1}}{\phi_{2}}=\exp \left(\frac{E_{a_{1}}-E_{a_{2}}}{\mathrm{RT}}\right)$,

In this expression, the subscripts 1 and 2 correspond to the two detections bands. The ratio $R$ does not depend on the number of excited molecules $N$, nor on the intensity of the laser excitation $I_{0}$. It is therefore particularly well suited for measuring the temperature of droplets whose size, trajectory, and concentration of dye cannot be controlled in a spray. The sensitivity of the technique corresponds to the difference $\left(E_{a_{1}}-E_{a_{2}}\right)$ between the two carefully selected spectral bands. In a previous study conducted in a spray, Labergue et al. (2010) used KR for measuring the droplet 
temperature with the spectral bands [ $535 \mathrm{~nm}-545 \mathrm{~nm}]$ and $[615 \mathrm{~nm}-750 \mathrm{~nm}]$. They reported a temperature sensitivity of the ratio of about $0.9 \% /{ }^{\circ} \mathrm{C}$. Using only one spectral band, the technique developed in the present study has a much higher temperature sensitivity with the same dye. With values of $E_{a}$ around $19-20 \mathrm{~kJ} / \mathrm{mol}$, the temperature sensitivity reaches about $2.6 \% /{ }^{\circ} \mathrm{C}$ in the range $20-80 \mathrm{C}$. To obtain comparable values of sensibility using a ratiometric method, it is necessary to use a mixture of two dyes (Chaze et al. 2016). However, these dyes need to have absorption and emission spectra that are well separated in wavelength to avoid any spectral conflict and the practical implementation of the method usually requires extensive preliminary tests.

\section{Application to droplets in polydisperse sprays}

\subsection{Preliminary tests in sprays under isothermal conditions}

To test the measurement method, a spray configuration which has been already examined in earlier studies (Labergue et al. 2010, 2012; Rimbert and Castanet 2010) was chosen, as it illustrates some of the problems that can be encountered in measuring the temperature in polydisperse sprays with the intensity of the fluorescence signal and the two-color laser-induced fluorescence technique. A full cone spray is generated by a pressure swirled atomizer (Danfoss oil nozzle, angle $30^{\circ}$ type $\mathrm{S}, 0.50 \mathrm{USgal} / \mathrm{h}, 1.87 \mathrm{~kg} / \mathrm{h}$ ) (Fig. 7). Before entering the nozzle, the liquid flows across a heat exchanger, which allows to control the liquid temperature that is injected. A K-type thermocouple inserted in the inlet pipe of the spray nozzle is used to determine the liquid injection temperature. The spraying system is also mounted on translations stages to move the spray relatively to the optics and perform measurements at different positions. The size and velocity distributions of the droplets can be found in the study by Labergue et al. (2012) who used the well-established Phase Doppler technique to obtain joint size/velocity PDFs. An increase in the injection pressure from 2 bar to 6.5 bar induces a decrease in the droplet mean diameter $D_{10}$ from $40 \mu \mathrm{m}$ to $20 \mu \mathrm{m}$, while the droplet density increases from $10^{3}$ to $5.10^{4}$ particles per $\mathrm{cm}^{3}$ at $z=13 \mathrm{~mm}$ downstream of the injector outlet (Figs. 5 and 6 in Labergue et al. 2012).

Using the same spraying system, Labergue et al. (2010) observed a significant distortion of the fluorescence spectrum when changing the injection pressure and thus the droplet size and local density (Fig. 6 in Labergue et al. 2010). They described for the first time, an effect apparently related to the size of the droplets, which is capable to alter the fluorescence spectrum and the ratio between signal intensities collected on two separated spectral bands. Later, Labergue et al. (2012) highlighted the importance of reducing the depth of field of the detection optics to reduce this effect. In a spray, multiple light scattering by the droplets can be significant, especially when the injection pressure and thus the density of the droplets are increased. While the laser beam has typically a diameter of a few hundred microns in the vicinity of the probe volume, light scattering considerably enlarges the region of the fluorescence excitation. (a)

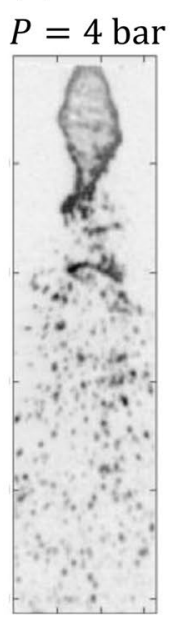

(b)

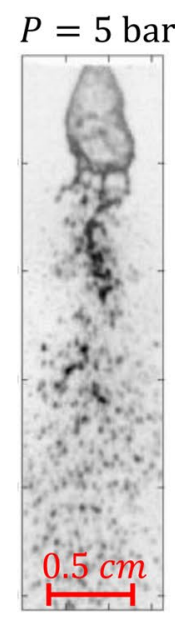

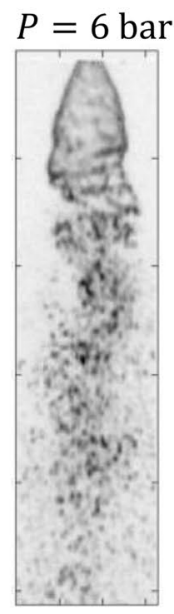

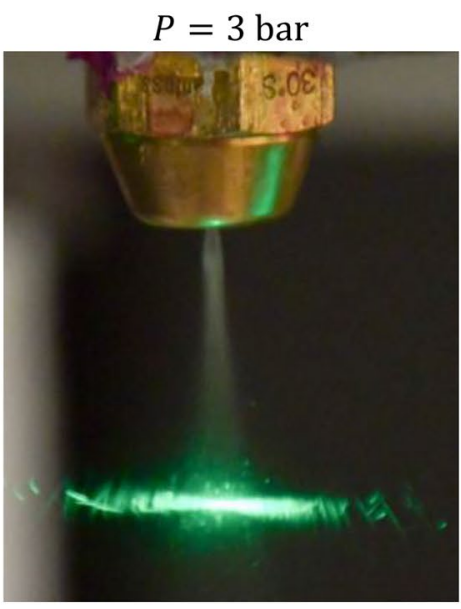

(c)

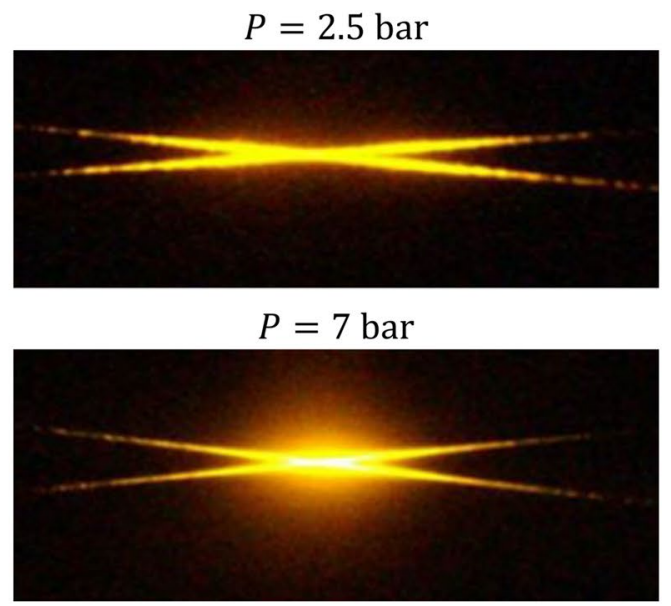

Fig. 7 Images of the spray at different injection pressures. a: Shadow images taken by Rimbert and Castanet (2010), b: Illumination with a focalized laser beam at $490 \mathrm{~nm}$, c: Fluorescence field induced by two laser beams crossing each other in a configuration of Laser Doppler
Velocimetry. Images extracted from Labergue et al. (2012). Photographs of the spray are showing evidence of multiple light scattering by the droplets in (b) and (c) 
Droplets normally localized well outside the laser beam emit a substantial amount of parasitic fluorescence because of laser light scattering (Fig. 7c). Although most of this fluorescence originates from regions outside of the focal plane of the collection optics, effects on the fluorescence signal can be significant. The detection system collects light originating from a region that has a significant depth on both sides of the focal plane (typically several $\mathrm{mm}$ ) as illustrated for similar optical systems in Fig. 13 in Labergue et al. (2012). Figure 8 partially reproduces results from Labergue et al. (2012) where they used for the collection of the LIF signal either an achromatic doublet $(f=310 \mathrm{~mm})$ or a long-distance microscope (Questar QM-100) with a rather small depth of field (about $100 \mu \mathrm{m}$ ). Figure 8 shows that the fluorescence ratio $R$ defined at Eq. (9) and calculated for KR on the spectral bands [535 nm-545 nm] and [615 nm-750 nm] varies considerably with the considered optical system. When the injection pressure is increased from 3 bar to $6.5 \mathrm{bar}$, the variations in the ratio $R$ are reduced with the long-distance microscope which removes more efficiently the out-of-field fluorescence (Fig. 13 in Labergue et al. 2012). Nevertheless, converted into temperature, the remaining variations in $R$ still correspond to a bias of more than $10 \mathrm{C}$. The front lens used for collecting the signal in the present study has the same deficiencies for measuring the droplet temperature with the 2cLIF technique, although it is an achromatic doublet. As illustrated in Fig. 8, the ratio $R$ varies within a significant range when using the same spectral bands as Labergue et al. (2012). Given the difficulties in eliminating this measurement bias, Labergue et al. (2012) developed an empirical method of correction using a third spectral band

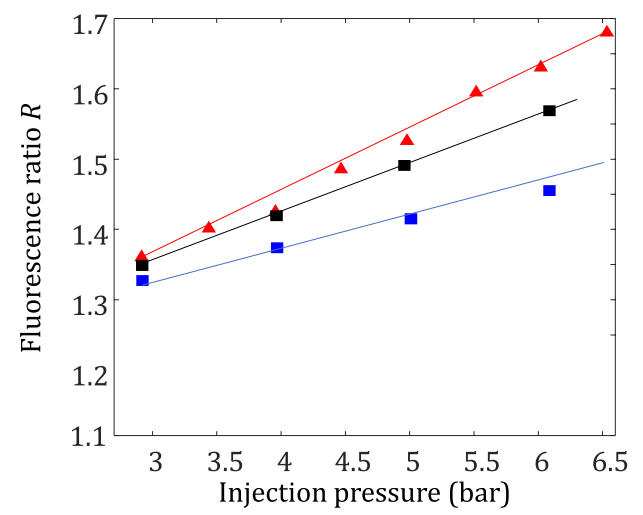

- Achromatic doublet $f=90 \mathrm{~mm}$ (present study)

— Achromatic doublet $f=310 \mathrm{~mm}$ (Labergue et al. 2012)

- Long distance microscope (Labergue et al. 2012)

Fig. 8 Evolution of the fluorescence ratio $R$ as a function of the injection pressure for different detection optics. The fluorescence signal is detected on the spectral bands [535 nm-545 nm] and [615 nm-675 nm] downstream of the nozzle outlet at $z=13 \mathrm{~mm}$. Liquid is injected at ambient room temperature $\left(T_{\mathrm{inj}} \approx 20^{\circ} \mathrm{C}\right)$ of detection. Although many measurement biases seem to be effectively corrected, it cannot be ensured that all are completely removed due to the lack of a conceptual basis for these corrections. Additional effects can be suspected to affect the fluorescence ratio. Fluorescence reabsorption preferentially concerns the green region of the fluorescence emission spectrum of RhB and KR below about $620 \mathrm{~nm}$. In several situations, stimulated emission of the dye molecules was observed because of Morphology-Dependent Resonances (MDRs) within the droplets (Wolff et al. 2007; Perrin et al. 2015).

Preliminary tests have been performed to verify that lifetime-based measurements are not affected by the problems discussed above. In a first step, the injection pressure was increased from 2 to 6 bar while the injection temperature was fixed at $19 \mathrm{C}$. Then, lifetime measurements were performed at $z=13 \mathrm{~mm}$, which is the same downstream position as the data displayed in Fig. 8. The evolution of the fluorescent lifetime as a function of the injection pressure is presented in Fig. 9. Whatever the fluorescent dye and the spectral band of detection are, the fluorescence lifetime $\tau$ is not changing with the injection pressure. Variations in $\tau$ do not exceed $\pm 1 \%$, which corresponds to $\pm 0.4^{\circ} \mathrm{C}$ using the activation energy $E_{a}$ determined for $\mathrm{RhB}$ or $\mathrm{KR}$ in the spectral band [ $630 \mathrm{~nm}-650 \mathrm{~nm}$ ] (Table 1). Variations in $\tau$ are simply related to the inherent uncertainties of the measurements. At this distance from the nozzle, the fragmentation of the liquid has already occurred (Fig. 7a), but the cooling of the droplet is still very limited.

In a second step, the injection temperature was gradually increased, and the fluorescence lifetime was measured for $\mathrm{KR}$ in the spectral band $[630 \mathrm{~nm}-650 \mathrm{~nm}]$ at $z=10 \mathrm{~mm}$. The results were then compared to measurements performed at the same temperature in a cell and in a monodisperse droplet stream where the droplet size is about $150^{\circ} \mathrm{C} \mu \mathrm{m}$ (much larger than the mean diameter $D_{10}$ in the spray). The results, presented in Fig. 10 for $T_{\text {inj }}=20$ and $T_{\text {inj }}=50{ }^{\circ} \mathrm{C}$, reveal that the fluorescence decay practically does not change in all these situations. A lifetime difference of about $0.015 \mathrm{~ns}$ is observed for both $T_{\text {inj }}=20^{\circ} \mathrm{C}$ and $T_{\text {inj }}=50^{\circ} \mathrm{C}$. Such a small difference, corresponding to a bit more than $1^{\circ} \mathrm{C}$, can be explained by a deviation in the liquid temperature, despite the extra care taken to achieve the same temperature in the spray, the monodisperse droplet stream, and the cell. Finally, the main difference between the cell and the spray appears to be in the noise contribution, which is higher in the spray signal. It obviously takes a longer time to acquire as many photons in a spray as in a cell due to a lower liquid volume fraction in the probed volume. In a spray, the liquid fraction rapidly reduces downstream of the nozzle to reach only a few percent at $z=13 \mathrm{~mm}$. As long as the noise level is known, the noise contribution can be taken into account in the fitting model of the fluorescence decay. Nonetheless, a high level 
(a)

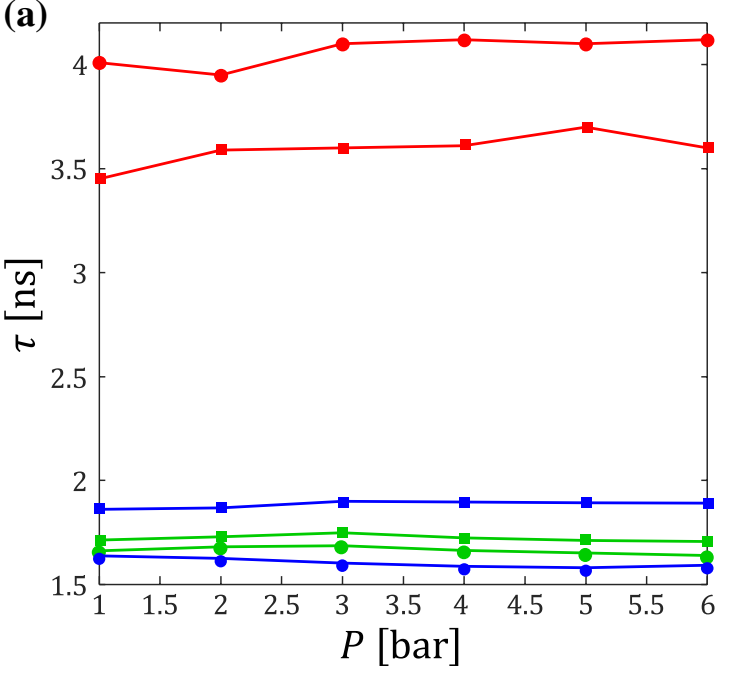

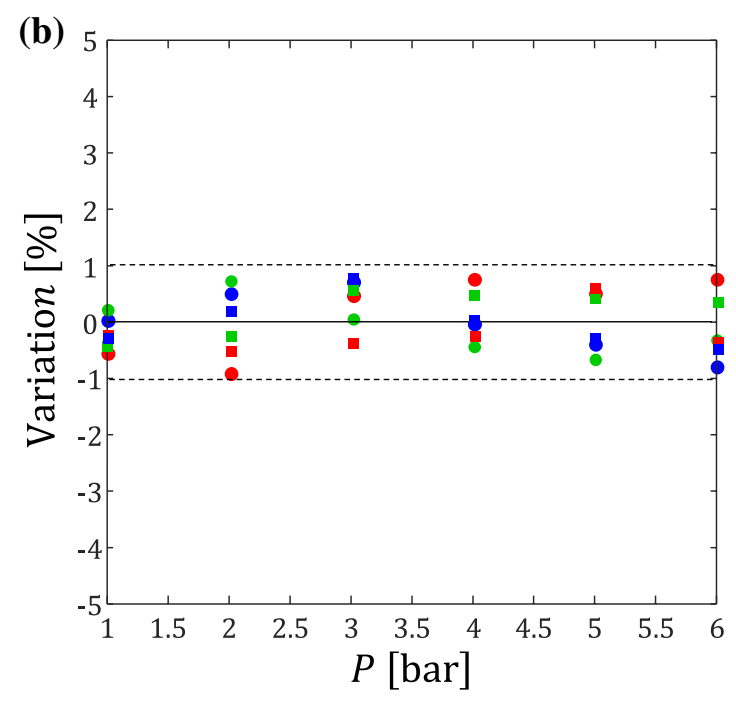

\begin{tabular}{|lll|}
\hline- Rh6G & $-\mathrm{RhB}$ & $-\mathrm{KR}$ \\
$-[630 \mathrm{~nm}-650 \mathrm{~nm}]$ & $-[630 \mathrm{~nm}-650 \mathrm{~nm}]$ & $-[630 \mathrm{~nm}-650 \mathrm{~nm}]$ \\
$\cdot[540 \mathrm{~nm}-560 \mathrm{~nm}]$ & $\cdot[540 \mathrm{~nm}-560 \mathrm{~nm}]$ & $\cdot[540 \mathrm{~nm}-560 \mathrm{~nm}]$ \\
\hline
\end{tabular}

Fig. 9 Evolution of the fluorescence lifetime in function of the injection pressure for different dyes and spectral bands of detection. Measurements performed at $z=13 \mathrm{~mm}$ and an injection temperature $T_{\text {inj }}=19^{\circ} \mathrm{C}$

Table 1 Activation energy $E_{\text {a }}$ calculated either from the fluorescence lifetime or from the fluorescence intensity measured using a CW laser at $532 \mathrm{~nm}$

\begin{tabular}{lll}
\hline Spectral bands & $\begin{array}{l}\text { Lifetime measurement } E_{a} \\
(\mathrm{~kJ} / \mathrm{mol})\end{array}$ & $\begin{array}{l}\text { Intensity meas- } \\
\text { urement } E_{a}(\mathrm{~kJ} / \\
\text { mol })\end{array}$ \\
\hline $\begin{array}{l}\text { Activation energy } E_{a} \text { for rhodamine B }(\mathrm{RhB}) \\
\text { 525-535 }\end{array}$ & $13.8 \pm 0.6$ & $10.5 \pm 0.5$ \\
$540-560$ & $18.7 \pm 0.5$ & $18.6 \pm 0.2$ \\
$570-590$ & $19.3 \pm 0.6$ & $22 \pm 0.3$ \\
$630-650$ & $19.8 \pm 0.48$ & $19.5 \pm 0.6$ \\
Activation energy $E_{a}$ for kiton red $(\mathrm{KR})$ & & - \\
525-535 & $0.043 \pm 0.2$ & $12.8 \pm 0.6$ \\
$540-560$ & $12 \pm 0.5$ & $22.5 \pm 0.2$ \\
570-590 & $19.4 \pm 0.6$ & $20.1 \pm 0.4$ \\
$630-650$ & $18.7 \pm 0.4$ & $1 \pm 0.02$ \\
Activation energy $E_{a}$ for rhodamine 6G $(\mathrm{Rh6G})$ & & $0.08 \pm 0.02$ \\
540-560 & $0.28 \pm 0.06$ & \\
630-650 & $0.16 \pm 0.06$ & \\
\hline
\end{tabular}

$\mathrm{RhB}$ (a), KR (b) and Rh6G (c) are at a concentration of $10^{-5} \mathrm{M}$ in the liquid cell of noise will reduce the dynamic range of the measurements and thus the time interval useable for the fitting of $\tau$. In Fig. 10b, the dynamic range decreases from about $3.5 \mathrm{dec}-$ ades in the cell to slightly less than 3 decades in the spray. This decrease is moderate enough that the accuracy of the fitting is not noticeably degraded. A low noise is decisive to maintain a high SNR and therefore a wide dynamic range even in the low-density regions of the spray. From these preliminary tests, it seems that the temperature measurements using the TCSPC technique are not affected by any bias, as opposed to intensity-based measurements using the 2cLIF method. 

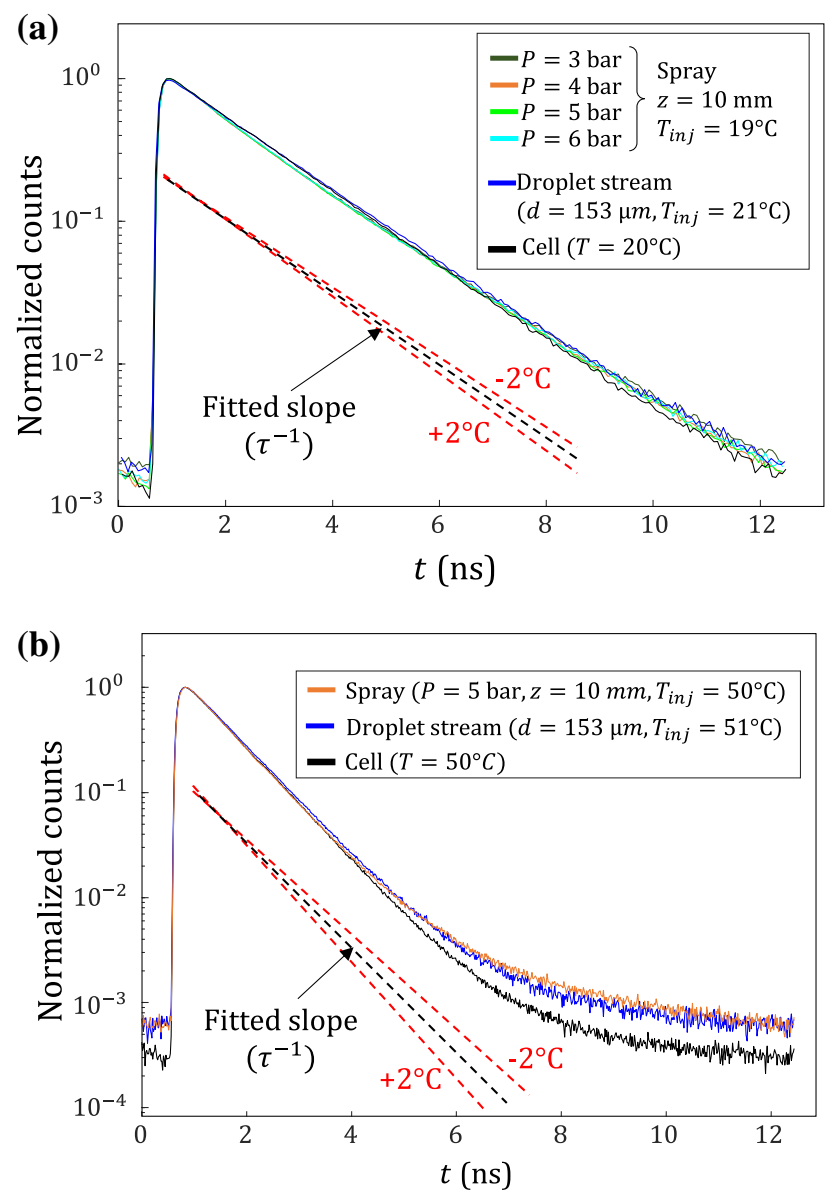

Fig. 10 Fluorescent decay of KR in a spray ( $z=13 \mathrm{~mm}, P=5 \mathrm{bar}$ ), in a droplet stream (at $z=10 \mathrm{~mm}$ ) and in a cell for two liquid temperatures: $T=20^{\circ} \mathrm{C}$ (a) and $T=50^{\circ} \mathrm{C}(\mathbf{b})$. The slopes for a temperature change of $\pm 2^{\circ} \mathrm{C}$ are provided to illustrate that the differences in fluorescence lifetime between the observations is on the order of $\pm 1^{\circ} \mathrm{C}$

\subsection{Application to study the cooling of droplets in a heated spray}

The measurement technique was then used to characterize the liquid cooling in the spray for different injection temperatures and an injection pressure of $P=5$ bar. Figure 11 presents the evolution of the mean temperature of the droplets on the spray axis for $T_{\mathrm{inj}}=19^{\circ} \mathrm{C}, 44^{\circ} \mathrm{C}$ and $60^{\circ} \mathrm{C}$. Due to evaporation, droplets injected at room temperature (or even slightly lower) can be cooled down (Depredurand et al. 2010). While the cooling rate is dependent on the size, velocity, and initial temperature of the droplets, their final equilibrium temperature depends basically on the ambient air temperature $T_{\mathrm{amb}}$ and the relative humidity $\mathrm{RH}$. It corresponds to the wet-bulb temperature $T_{\mathrm{wb}}$. At $\mathrm{RH}=100 \%$, the wet-bulb temperature is equal to the air temperature (drybulb temperature); at lower humidity the wet-bulb temperature is lower than dry-bulb temperature because of evaporative cooling. Under the experimental conditions in Fig. 11,

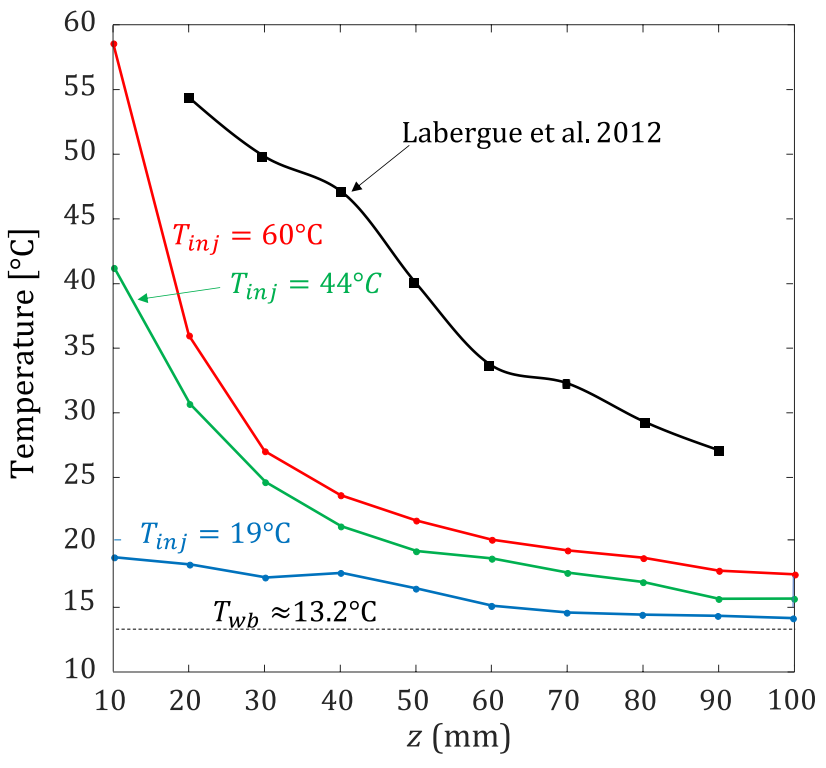

Fig. 11 Evolution of the droplet temperature measured in the centerline of the spray for different injection temperature $(P=5$ bar). Comparison to the data measurements from Labergue et al. (2012) at $P=4.5$ bar

$T_{\text {amb }}=19^{\circ} \mathrm{C}$ and $\mathrm{RH}=52 \%$, the wet-bulb temperature can be valuated at $T_{\mathrm{wb}}=13.2^{\circ} \mathrm{C}$ (Stull 2011). In Fig. 11, measurements seem to converge toward this theoretical limit for the case $T_{\text {inj }}=19^{\circ} \mathrm{C}$. For $T_{\text {inj }}=44^{\circ} \mathrm{C}$ and $60^{\circ} \mathrm{C}$, the droplets have not reached their thermal equilibrium at $z=100 \mathrm{~mm}$. It should be mentioned that the spray was injected into a large room in which air is constantly refreshed. However, local humidity inside the spray is surely higher than in the ambient, due to vaporization of the droplets. A relative humidity of $88 \%$ would be necessary to explain an equilibrium temperature of $17.5^{\circ} \mathrm{C}$, corresponding to the smallest temperature observed for $T_{\text {inj }}=60^{\circ} \mathrm{C}$, but the droplets will further cool down after $z=100 \mathrm{~mm}$. A comparison is also possible with measurements carried out by Labergue et al. (2012) using the intensity of the fluorescence signal, collected on three spectral bands. Measurements obtained by Labergue et al. (2012) for $T_{\mathrm{inj}}=51^{\circ} \mathrm{C}$ and $P=4.5 \mathrm{bar}$ are also added in Fig. 11. An important difference can be observed between both measurement techniques. Clearly, the cooling rate is lower and does not follow the same time evolution. This can hardly be explained by differences in the injection conditions ( $P=4.5$ bar against $P=5$ bar), nor by the ambient conditions (unfortunately, Labergue et al. (2012) provide no information about the relative humidity or about the ambient temperature). More likely, intrinsic biases are affecting the intensity-based measurements performed by Labergue et al. (2012). As acknowledged by the authors themselves, the smallest droplets may have been discriminated against the biggest ones. The fluorescence signal is detected by 
Labergue et al. (2012) by means of three photomultiplier tubes with a threshold level fixed significantly above the noise level of the channels of detection to detect the droplets on an individual basis. If a sequence has more than ten consecutive samples above this threshold, it is considered coming from droplets; otherwise, the sequence is fully rejected. Obviously, this condition is more difficult to fulfill for very small droplets.

The fluorescence signal emitted by a droplet varies roughly with $D^{3}, D$ being the droplet diameter. However, in these measurements, the dynamic range of the PMT detectors (ratio of the maximum signal and the noise level) was hardly greater than 15-20, making it difficult to study a droplet population whose size ranges over more than a 2.5:1 ratio. Considering that the high voltages of the PMTs were adjusted so as to detect the largest droplets without any saturation of the signal level, many of the small droplets could simply not be detected and monitored by the measurement system used by Labergue et al. (2012). This issue is not encountered in the case of the current lifetime-based measurement technique which operates on single photons with no distinction whether they originate from a small or a large droplet. Given that the biggest droplets of the size distribution require more time to cool down and reach the equilibrium temperature, the size bias affecting intensitybased measurements by Labergue et al. (2012) provides an explanation for the differences in cooling rate observed for the two techniques in Fig. 11. In the TCSPC technique, a constant fraction discriminator is used to extract precise timing information from the electrical detector pulses. While it can significantly modify the photon count rate, photons that are eventually rejected are not correlated with the droplet size. Therefore, we believe that the temperature measured using TCSPC is very close to the volume average temperature of the liquid phase without any weighting of this average by the size distribution of the spray.

\subsection{Discussion on measurement biases and uncertainties}

A detailed analysis of the errors has been conducted to quantify the accuracy of these temperature measurements in spray. Basically, there are two main sources of errors in the droplet temperature measurements: (i) errors resulting from the temperature calibration of the fluorescence lifetime and (ii) errors made in the lifetime measurements in the spray. Both contributions are examined in detail in the following:

i) Error originating from the temperature calibration

Any error in the parameters $A$ and $E_{a}$ of Eq. (8) will result in a temperature error when the lifetime is converted into temperature after the measurements. A Monte Carlo method of error propagation was implemented to assess the effect on the measured temperature accuracy. The method consists of repeated calculations of quantities $A$ and $E_{a}$, each time randomly varying the position of the points on the calibration curve within their stated limits of precision. During the calibration in a cell (Sect. 3), the liquid temperature is measured with an uncertainty of $\pm 0.2^{\circ} \mathrm{C}$ using a thermocouple, while the uncertainty of the lifetime can be determined from the Jacobian of the minimization function $\chi^{2}$. For any point $k$ of the calibration curve, the lifetime $\tau_{k}$ and the temperature $T_{k}$ are randomly generated considering normal distributions with a standard deviation mirroring the uncertainties on these quantities. Coefficients $A$ and $E_{a}$ are estimated by a linear regression of $\ln \left(\tau_{k}\right)$ versus $1 / T_{k}$. Repeating this random sampling of the calibration points many times (typically $n=10,000$ ), it is possible to evaluate the means and the standard deviations of the coefficients $A$ and $E_{a}$. Resulting confidence intervals are indicated in the second column of Table 1 for the activation energy $E_{a}$. They correspond to $\pm 2 \sigma_{E_{a}}$, where $\sigma_{E_{a}}$ is the standard deviation of $E_{a}$. Finally, for the $i^{\text {th }}$ of the random samplings, estimated values $\left(E_{a_{i}}, A_{i}\right)$ can be used to convert a chosen lifetime $\tau_{m}$ into a temperature $T_{m, i}$. The distribution of the temperatures $T_{m, i}$ calculated from many random samplings shows the effects of calibration errors on the measured temperature $T_{m}$. Figure 12 displays the standard deviation $\sigma_{T_{m}}$ while $T_{m}$ is ranging from $15^{\circ} \mathrm{C}$ to $60^{\circ} \mathrm{C}$ for the measurement configuration used in the spray. Uncertainties on the calibration coefficients $A$ and $E_{a}$ yield an uncertainty on the temperature that can be evaluated as $\pm 2 \sigma_{T_{m}}$. This latter can reach $\pm 1 \mathrm{t}$ $T_{m}=60^{\circ} \mathrm{C}$, but on the average, it is close to $\pm 0.6 \mathrm{C}$ for the measurements reported in Fig. 11. The uncertainty is larger on the edges of the temperature range which was considered

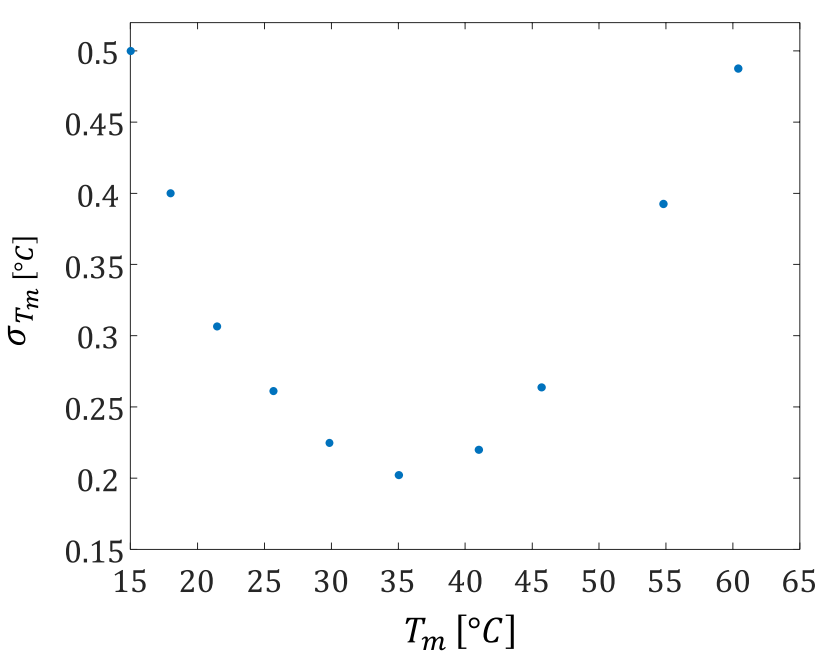

Fig. 12 Evolution of the standard deviation in function of the temperature $\mathrm{Tm}$. The uncertainty is the lowest around $40^{\circ} \mathrm{C}$ in the middle of the temperature calibration range 

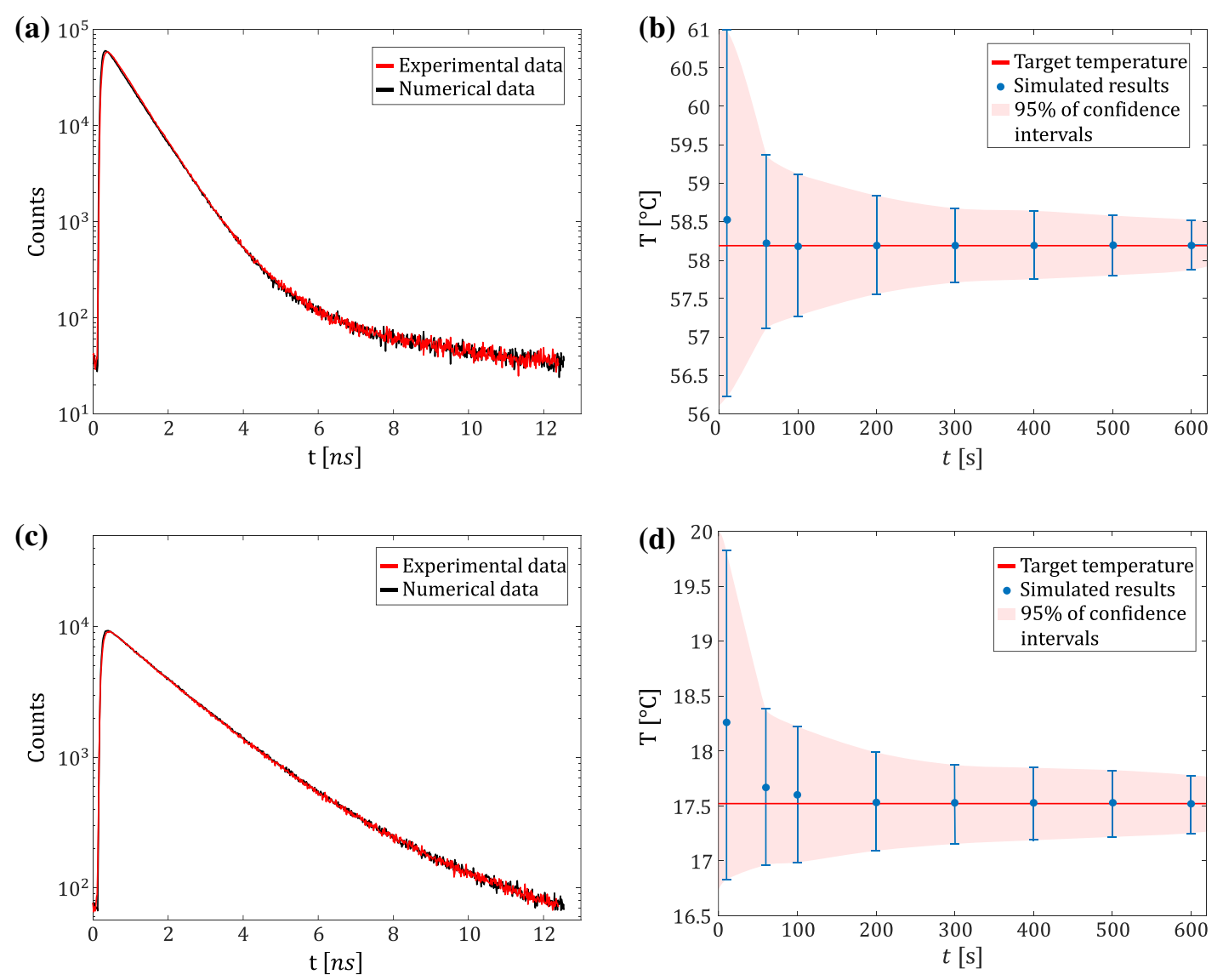

Fig. 13 Fluorescence decays corresponding to the measurement data in Fig. 11 at (a) $z=10 \mathrm{~mm}$ and $T_{\mathrm{inj}}=60^{\circ} \mathrm{C}$ (acquisition time: $\left.300 \mathrm{~s}, 8508 \mathrm{Cps}, a=0.01311, \tau_{1}=1.548 \mathrm{~ns}, \tau_{2}=0.697 \mathrm{~ns}\right)$ and (c) $z=100 \mathrm{~mm}$ and $T_{\mathrm{inj}}=60^{\circ} \mathrm{C}$ (acquisition time: $400 \mathrm{~s}, 2700 \mathrm{Cps}$,

for the calibration $\left[20-60^{\circ} \mathrm{C}\right]$. A wider range of temperature in the calibrations may substantially reduce this uncertainty.

ii) Error made in the measurement of the fluorescence lifetime

In a spray, the small volume fraction of liquid in the probe volume reduces the fluorescence signal compared to measurements in a liquid cell. A lower signal-to-noise ratio (SNR) is therefore encountered. In Fig. 11, the lowest count rate obtained at $z=100 \mathrm{~mm}$ is above $2700 \mathrm{Cps}$. This is usually one order of magnitude less than at the nozzle outlet and two orders of magnitude less than in a liquid cell, but still far above the noise (about $60 \mathrm{Cps}$ ). To estimate measurement errors, here again, a Monte Carlo simulation method was implemented. For a given value of the lifetime $\tau$, it is possible to generate synthetic histograms of the photon arrival times while taking into account the photon count rate, the acquisition time, the noise level, the IRF, and the time-channel width. In Figs. 13a and c, the Monte

$\left.a=0.8919, \tau_{1}=1.653 \mathrm{~ns}, \tau_{2}=3.275 \mathrm{~ns}\right)$. The $95 \%$ confidence intervals reported in (b) and (d) are calculated for the same cases using Monte Carlo simulations. Each point is the result of 1000 synthetic fluorescence decays equivalent to the ones displayed in (a) and (c)

Carlo-based simulation of the fluorescence decay is validated against experimental results for two positions in the spray at $T_{\mathrm{inj}}=60^{\circ} \mathrm{C}$, namely $z=10 \mathrm{~mm}$ and $z=100 \mathrm{~mm}$. An identical number of photons as those in the experimental decays are randomly distributed in time from a probability distribution that allows to mimic the characteristics of the measurement system. This approach offers a simple way to figure out how many photons are necessary for reliable fluorescence lifetime measurements in the spray. Hence, the acquisition time was changed from $10 \mathrm{~s}$ to $10 \mathrm{~min}$ while maintaining the values of the lifetime, the count rate $(8508$ Cps at $z=10 \mathrm{~mm}$ and $2700 \mathrm{Cps}$ at $z=100 \mathrm{~mm}$ ) as well as the noise level $(60 \mathrm{Cps})$, and for each acquisition time that was tested, 1000 synthetic histograms were generated and analyzed to estimate the lifetime by minimizing $\chi^{2}$ and the temperature. The standard deviation $\sigma_{T}$ of the obtained temperature indicates the uncertainty of the measurements. Figure $13 \mathrm{~b}$ and $\mathrm{d}$ shows the evolution of $\sigma_{T}$ as a function of the acquisition time for the same measurement conditions as in $z=10 \mathrm{~mm}$ and $z=100 \mathrm{~mm}$. Even far from the nozzle 
outlet at $z=100 \mathrm{~mm}$, an accuracy of $\pm 0.6^{\circ} \mathrm{C}$ can be reached provided that the acquisition time exceeds $200 \mathrm{~s}$ in order to achieve a good statistical convergence. It should be noted that the measurements in Fig. 11 were completed in the present study with acquisition times of about $300 \mathrm{~s}$.

For completeness, it is interesting to consider different phenomena that can affect the fluorescence signal and to discuss their possible effect on the lifetime measurement.

- Multiple scattering by the droplets: Light scattering can induce a longer decay of the fluorescence signal under two conditions:

- Fluorescence photons which are scattered several times but ultimately returns into the detector.

- Laser photons which are scattered several times but arrive in the measurement volume to induce the fluorescence viewed by the detector.

To obtain a significant variation in the decay time of 0.1 ns (which corresponds to an error of the order of $1-2^{\circ} \mathrm{C}$ on the temperature), a significant part of the detected photons should have been delayed due to an extra path lengthened by at least $3 \mathrm{~cm}$ (either in the way to the detector or to the measurement volume) which is a long distance compared to the spray diameter. Presence of multiple scattering can be observed by a halo in Fig. 7, but the halo size is only of a few millimeters. In principle, when photons undergo many random scattering interactions, they eventually become uncorrelated in time with the laser pulse. They only accounts for noise in the histogram. They reduce the signal dynamic but do not change the rate of the fluorescence decay.

-Morphology-dependent resonances: MDRs can induce a stimulated emission of the dye. The droplet behaves like a dye laser pumped by the excitation laser (here the femtosecond laser). The use of an ultrashort laser (of the order of $150 \mathrm{fs}$ ) limits the development of MDR modes as the light cannot make many turns around the droplet surface. At the end of each laser pulse, the pumping of the optical cavity ends. Consequently, the signal decay recorded by TCSPC is observed over a period of several ns without any lasing of the dye in the droplets.

Saturated excitation of the fluorescence: When nanosecond-pulsed lasers are used with a high radiance, a loss of temperature sensitivity was observed from some fluorescent dyes like RhB and KR (Chaze et al. 2016). Even though a saturation of the fluorescence can occur when using ultrashort laser pulse in the present measurements, this has no consequence on TCSPC measurements. Saturation only changes the number of molecules placed in the excited state during the femtosecond laser pulse. The decay rate measured after the pulses, is unchanged.

Fluorescence reabsorption: For some detection bands, fluorescence can be reabsorbed. This reabsorption is strongly detrimental for $2 \mathrm{cLIF}$ measurements when it affects one of the two bands of detection. For lifetime measurements, it is also a problem since the lifetime and its sensitivity to temperature vary with the wavelength. Special care was thus taken in the present study to detect the fluorescence signal in the band [630 nm-650 nm], which is neither reabsorbed by KR or RhB. Reabsorption can induce a secondary fluorescence emission, but its possible effect on the signal decay is not noticeable in the measurements (Fig. 10).

Increase in the concentration of the fluorescence dye: When evaporation is considered, the concentration of the fluorescence dye increases with time which can modify the fluorescence lifetime. Measurements reveal that the lifetimes of RhB and KR are constant for concentrations below $10^{-3}$. For higher concentrations, the lifetime decreases abruptly due to energy transfers between fluorescent molecules (self-quenching). The spray measurements were performed at a concentration of $10^{-5} \mathrm{M}$ and $10^{-6} \mathrm{M}$. The volume of the droplets shall be divided by a factor of 100 and their size by a factor of 5 at least, so that an effect of self-quenching can arise. In the present study, evaporation is not so important as suggested by classical evaporation models (Abramzon and Sirignano 1989).

\section{Conclusion}

A new method for measuring the local mean temperature of the droplets in sprays was developed and tested. The method is based on the measurement of the fluorescence lifetime of certain dyes for which the fluorescence quantum yield is particularly sensitive to temperature such as rhodamine B and kiton red.

Preliminary calibration is carried out in a liquid cell to characterize the temperature evolution of the fluorescence lifetime for rhodamine B and kiton red. Although the decay law is based on first-order kinetics, in practice, fluorescence decays of rhodamine B and kiton red are more complex. Quenching processes and other environmental parameters lead to a double exponential decay behavior. This behavior is observed for the emission in the green region of the fluorescence spectrum at any temperature and at higher temperatures (typically above $40^{\circ} \mathrm{C}$ ) for the whole emission spectrum.

One of the major advantages of using the fluorescence lifetime is the fact that it is an absolute measurement, unlike the intensity, which is always relative. The fluorescence lifetime is an intrinsic molecular property, which is not affected 
by fluctuations in excitation source intensity or in the collections of the fluorescence signal. This makes all the difference when measuring the temperature in dense sprays also with two-color ratiometric techniques which are in principle quite robust and reliable for the investigations of two-phase flows. No bias in the measurements could be observed when changing the droplet size distribution contrary to previous $2 \mathrm{cLIF}$ measurements carried out under the same spray conditions. Some of the problems encountered by 2 cLIF can surely be circumvented by the SLIPI method (Mishra et al. 2016) which uses a structured laser illumination planar imaging to filter the fluorescence induced outside of the laser sheet by multiple light scattering. Nevertheless, SLIPI is not totally free of biases. For instance, it cannot correct for the fluorescence attenuation on the path between the laser plane and the detector which is function of the spray environment and can differently affect the detection bands used to calculate the fluorescence ratio.

Time-correlated single-photon counting (TCSPC) is particularly well suited to carry out temperature measurements because it is certainly the most sensitive approach for determining fluorescence lifetimes. A very high signal-tonoise ratio could be achieved under all the conditions which were tested in sprays. A dynamic range of about 3 decades for the decay curve can be retained in the spray, which is just slightly lower than in a liquid cell. This provides to the measurements an accuracy and robustness which is probably unrivaled among the other temperature measurement techniques available in sprays. The absolute error typically does not exceed $\pm 1^{\circ} \mathrm{C}$ in most cases and even reduces to about $\pm 0.5^{\circ} \mathrm{C}$ if any uncertainty affecting the calibration coefficients are put aside.

Although the technique is not suitable for measuring the temperature of individual droplets as opposed to intensitybased measurements (Labergue et al. 2012), it is possible to obtain the mean volume temperature of the liquid phase with no bias. Intensity-based measurements are usually limited by the dynamic range of their detectors, which are not able to detect with the same settings (gain voltage, laser power, etc.) individual droplets when they have very different size. This problem is not encountered with lifetime measurements and the TCSPC technique which operates on single photons regardless of their origin (large or small droplets).

Among the limitations of the technique, an acquisition time of a few minutes was necessary in our experimental spray conditions to ensure an optimal convergence of the measurement data and thus the highest accuracy. This limitation is mainly the result of the necessity to avoid any pile-up effect. The onset of the pile-up effect is not exactly at the same power value than in a liquid cell, so only in situ testing can provide its value.

\section{Appendix 1: Fitting of the fluorescence decay}

A nonlinear least squares analysis is used to test if the model is consistent with the data and estimate the parameters of the fluorescence decay model. Basically, parameters are varying until the goodness-of-fit parameter $\chi^{2}$ determined by:

$\chi^{2}=\frac{1}{n} \sum_{k=1}^{n} \frac{\left(N\left(t_{k}\right)-N_{c}\left(t_{k}\right)\right)^{2}}{N\left(t_{k}\right)}$,

tends to a minimum. In the above expression, $N\left(t_{k}\right)$ is the measured counts at time $t_{k}, N_{c}\left(t_{k}\right)$ is the modeled number of counts at time $t_{k}$, and $n$ is the number of data points. A good fit is characterized by a value of $\chi^{2}$ close to 1 and the weighted residual between the measured and calculated decay curves randomly distributed around 0 . To assess the quality of the fitting, a 95\% confidence interval for $\tau$ can be also constructed from the Jacobian of the objective function of the minimization (namely $\chi^{2}$ ).

In practice, the accuracy can be limited by the timing jitter of the detector and the electronic components. The time spread introduced by the components of the TCSPC system can be determined by evaluating the overall instrument response function (IRF). The IRF presented in blue in Figs. 14 and 15 was determined in a water cell filled with micrometer-sized glass beads and non-fluorescent dyes. Typically, the IRF has a 90 ps width at half maximum (FWHM) with an after-pulse around $2 \mathrm{~ns}$, which is typical of a hybrid PM. To account for this response, which is not infinitely short, the exponential decay is convoluted with the IRF to obtain the theoretical value of the photon count $N_{c}$ in Eq. (10). The convolution between (IRF) and the weighted sum of fluorescent decay paths yields to $N c(t)$ :

$N_{c}(t)=\operatorname{IRF}(t) * \sum_{i=1}^{2} a_{i} e^{-\frac{t}{\tau_{i}}}$

where $a_{2}=1-a_{1}$. The parameters $a_{i}$ and $\tau_{i}$ are then adjusted to minimize the value of $\chi$. The noise coming from the PM and the environment is also considered by adding "noise" photons uniformly distributed in time to the count value $N_{c}$. The noise is determined after turning off the laser after or before the measurements. Typically, in the dark, the count rate of the noise is about $60 \mathrm{Cps}$. Figure 14 shows the fluorescence decays of $\mathrm{KR}$ in a cuvette at $18.6^{\circ} \mathrm{C}$ for the spectral band [ $630 \mathrm{~nm}-650 \mathrm{~nm}]$. This decay is perfectly monoexponential (Eq. 2), and if this is assumed to be so, the lifetime can be determined with a very narrow confidence interval of $\pm 0.002 \mathrm{~ns}$. The same result for $\tau$ can be obtained using the biexponential model (Eq. 3), since the least square minimization of $\chi^{2}$ yields $a=0.9963, \tau_{1}=1.7652 \mathrm{~ns}$ and $\tau_{2}=1.7648 \mathrm{~ns}$. 

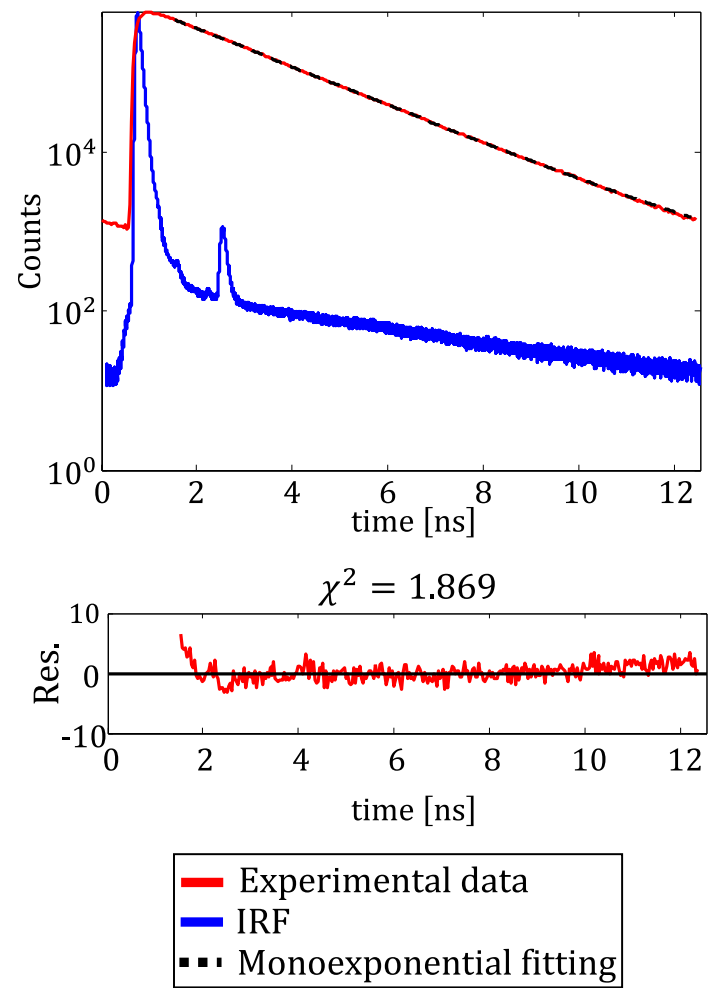

Fig. 14 Monoexponential fitting of the fluorescence decay of $\mathrm{KR}$ in a cell at $T=18.6^{\circ} \mathrm{C}\left(C=10^{-5} \mathrm{M}\right.$, spectral band: 630 $\mathrm{nm}-650 \mathrm{~nm}$ ). The lifetime value obtained by the least squares is to $\tau=1.765 \pm 0.002 \mathrm{~ns}$
For a higher temperature, for example close to $58{ }^{\circ} \mathrm{C}$ (Fig. 11), the fluorescence decay is no longer exactly a monoexponential function. The monoexponential fitting is not accurate since it yields a high value of $\chi^{2}$ and the residual is very large after about $4 \mathrm{~ns}$ (Fig. 15a). The quality of the fitting is correct if the time domain of the fitting is reduced to the initial linear region before $4 \mathrm{~ns}$, but this approach is not very rigorous since the result will depend on the chosen domain (Fig. 15b). By using the biexponential model, it is, however, possible to reproduce the decay over the entire time domain (Fig. 15c). In the following, experimental data will be fitted by a double exponential in order to obtain a reliable value for $\tau$ whatever the liquid temperature.

\section{Appendix 2: Measurement of the activation energy from intensity measurement}

An additional study was carried out by exposing the dye solutions in the cell to a continuous-wave Nd:YAG laser at $532 \mathrm{~nm}$. Emission spectra were recorded at different temperatures as shown in Fig. 16. For each wavelength of these spectra, an Arrhenus law can be used to fit the variation in the signal intensity as a function of temperature. The evolution of $E_{a}$ as a function of wavelength is also displayed in Fig. 16. For RhB and KR, it can be clearly observed that $E_{a}$ is decreasing in the green region of the emission spectra for wavelengths shorter than the emission peak, whereas in the case of Rh6G, $E_{a}$ is close to 0 over the whole emission
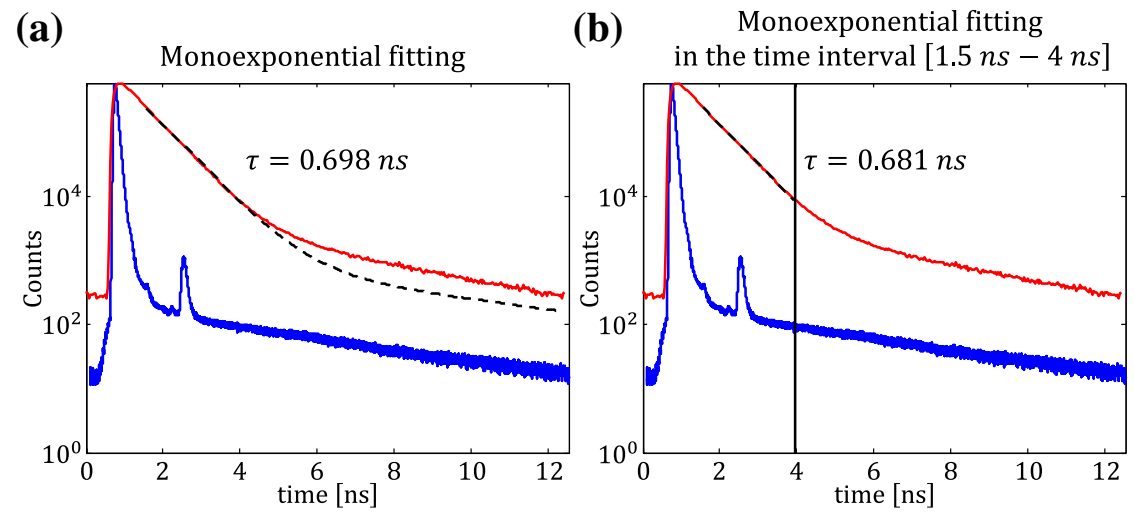

(c)

Biexponential fitting

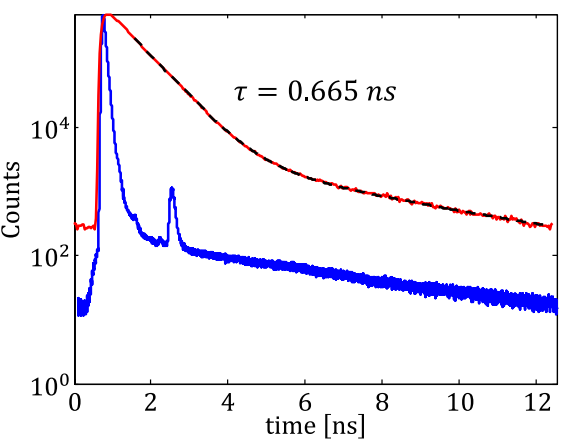

Experimental data $\quad$ IRF - - Fitting curve
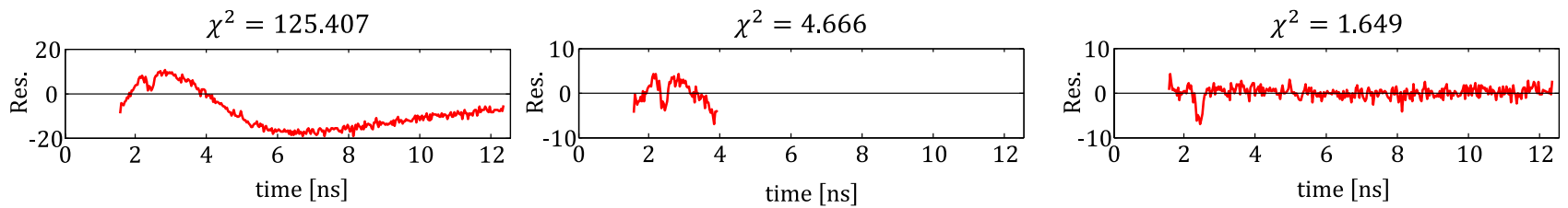

Fig. 15 Examination of different fitting approaches, a: Monoexponential fitting, $\mathbf{b}$ : Monoexponential fitting applied to a restricted time interval from $1.5 \mathrm{~ns}$ to $4 \mathrm{~ns}$, c: Biexponential fitting. Case of $\mathrm{KR}$ on the spectral band $[630 \mathrm{~nm}-650 \mathrm{~nm}]$ at $T=58^{\circ} \mathrm{C}$ and $\mathrm{C}_{\mathrm{KR}}=10^{-5} \mathrm{M}$.
The decay of the fluorescence exhibits a clear biexponential trend, which allows to reach a lower value of $\chi^{2}$ with the biexponential model in c 

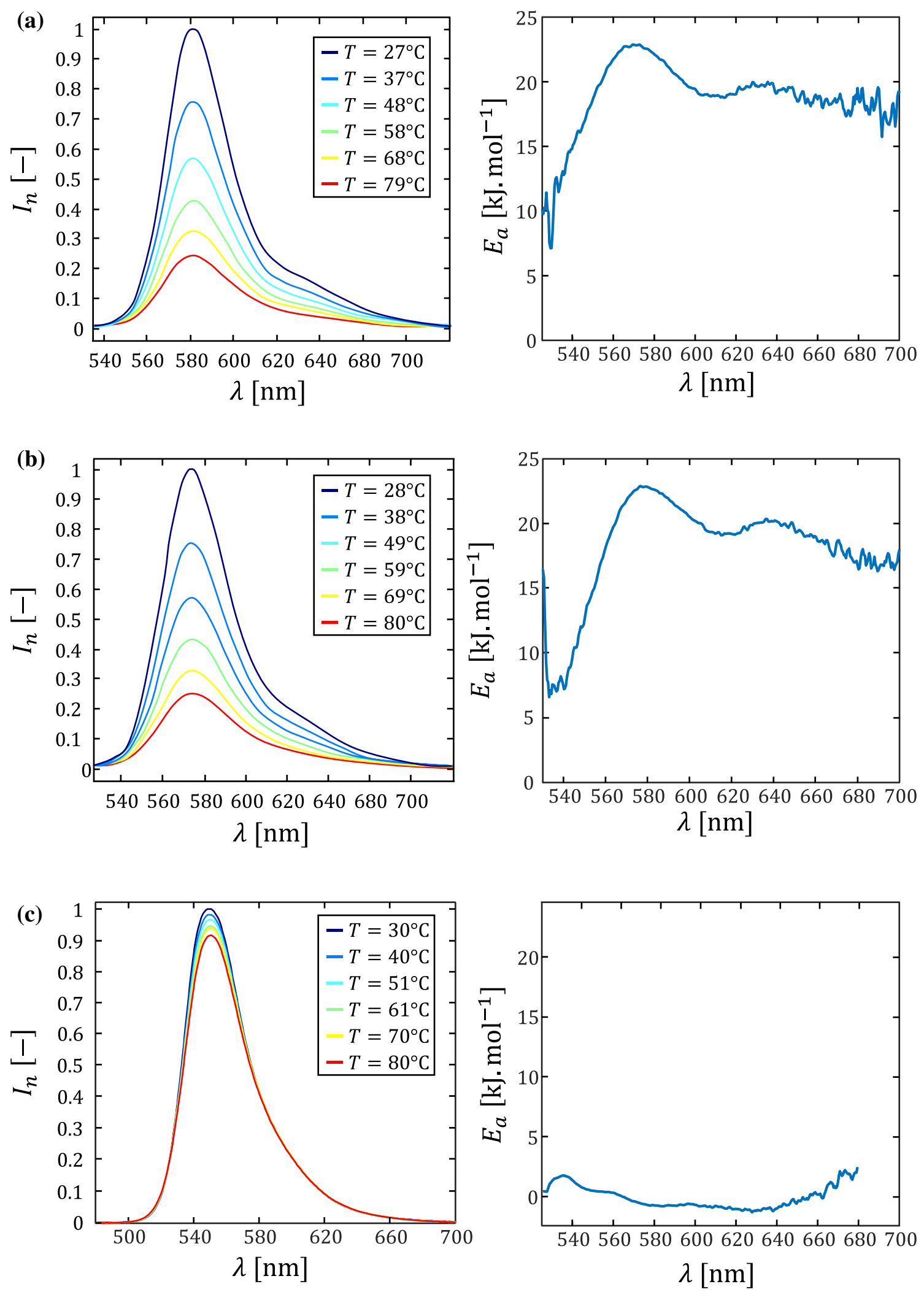

Fig. 16 Variation in the activation energy $E_{\mathrm{a}}$ in the Arrhenius equation (Eq. (8)) with the wavelength. Values assessed from emission spectra obtained at different temperatures in a liquid cell using a CW laser at $532 \mathrm{~nm}$ for a kiton red, b rhodamine B and $\mathbf{c}$ rhodamine $6 \mathrm{G}$ 
domain. Finally, the values of $E_{a}$ displayed in Fig. 16 are averaged over the spectral bands considered previously in the lifetime measurements and the results are added to Table 1.

Acknowledgements The authors would like to acknowledge the support of the China Scholarship Council under Program for Ph.D. Student Overseas Study Scholarship attributed to Mr. Liu, who was hosted by LEMTA at Universite de Lorraine, France. The authors also gratefully acknowledge the financial support of the ENERBATIN CPER program and the European FEDER program.

Author contributions Liu Yangpeng performed the measurements; Stiti Mehdi and Castanet Guillaume were involved in planning and supervised the work. Chaynes Hadrien was involved in the implementation of the optical system and the measurement methods. Liu Yangpeng, Stiti Mehdi, and Castanet Guillaume processed the experimental data, performed the analysis, drafted the manuscript, and designed the figures. Lemoine Fabrice and Wang Xishi provided critical feedback and helped shape the research. Castanet Guillaume conceived the original idea and was in charge of overall direction of the project with Lemoine Fabrice and Wang Xishi. All authors discussed the results and commented on the manuscript.

\section{References}

Abramzon B, Sirignano W (1989) Droplet vaporization model for spray combustion calculations. Int J Heat Mass Transf 32:1605-1618

Becker W (2005) Advanced time-correlated single photon counting techniques. Springer Ser. Chem. Phys.

Bennet MA, Richardson PR, Arlt J et al (2011) Optically trapped microsensors for microfluidic temperature measurement by fluorescence lifetime imaging microscopy. Lab Chip. https://doi.org/ 10.1039/c1lc20391f

Boens N, Qin W, Basarić N et al (2007) Fluorescence lifetime standards for time and frequency domain fluorescence spectroscopy. Anal Chem. https://doi.org/10.1021/ac062160k

Brutin D, Sobac B, Rigollet F, Le Niliot C (2011) Infrared visualization of thermal motion inside a sessile drop deposited onto a heated surface. Exp Therm Fluid Sci 35:521-530

Carns JC, Moncivais G, Brock JR (1990) Time-resolved raman spectroscopy from reacting optically levitated microdroplets. Appl Opt 29:2913-2918

Casadevall I, Solvas X, Srisa-Art M, Demello AJ, Edel JB (2010) Mapping of fluidic mixing in microdroplets with $1 \mu$ s time resolution using fluorescence lifetime imaging. Anal Chem 82:3950-3956

Casey KG, Quitevis EL (1988) Effect of solvent polarity on nonradiative processes in xanthene dyes: Rhodamine B in normal alcohols. J Phys Chem. https://doi.org/10.1021/j100334a023

Castanet G, Lavieille P, Lebouché M, Lemoine F (2003) Measurement of the temperature distribution within monodisperse combusting droplets in linear streams using two-color laser-induced fluorescence. Exp Fluids 35:563-571

Castanet G, Perrin L, Caballina O, Lemoine F (2016) Evaporation of closely-spaced interacting droplets arranged in a single row. Int $\mathbf{J}$ Therm Sci 93:788-802

Chaze W, Caballina O, Castanet G, Lemoine F (2016) The saturation of the fluorescence and its consequences for laser-induced fluorescence thermometry in liquid flows. Exp Fluids. https://doi.org/ $10.1007 / \mathrm{s} 00348-016-2142-8$
Chaze W, Caballina O, Castanet G, Lemoine F (2017) Spatially and temporally resolved measurements of the temperature inside droplets impinging on a hot solid surface. Exp Fluids. https://doi.org/ 10.1007/s00348-017-2375-1

Depredurand V, Castanet G, Lemoine F (2010) Heat and mass transfer in evaporating droplets in interaction: influence of the fuel. Int $\mathbf{J}$ Heat Mass Transf 53:3495-3502

Düwel I, Ge HW, Kronemayer H et al (2007) Experimental and numerical characterization of a turbulent spray flame. Proc Combust Inst 31(2):2247-2255

Förster T (1948) Zwischenmolekulare energiewanderung und fluoreszenz. Ann Phys. https://doi.org/10.1002/andp.19484370105

Frackowiak B, Tropea C (2010) Fluorescence modeling of droplets intersecting a focused laser beam. Opt Lett 35:1386

Hilaire MR, Mukherjee D, Troxler T, Gai F (2017) Solvent dependence of cyanoindole fluorescence lifetime. Chem Phys Lett 685:133-138

Kristoffersen AS, Erga SR, Hamre B, Frette Ø (2014) Testing fluorescence lifetime standards using two-photon excitation and timedomain instrumentation: Rhodamine B, coumarin 6 and lucifer yellow. J Fluoresc. https://doi.org/10.1007/s10895-014-1368-1

Labergue A, Deprédurand V, Delconte A et al (2010) New insight into two-color LIF thermometry applied to temperature measurements of droplets. Exp Fluids 49:547-556

Labergue A, Delconte A, Castanet G, Lemoine F (2012) Study of the droplet size effect coupled with the laser light scattering in sprays for two-color LIF thermometry measurements. Exp Fluids 52:1121-1132

Labergue A, Pena-Carillo J-D, Gradeck M, Lemoine F (2017) Combined three-color LIF-PDA measurements and infrared thermography applied to the study of the spray impingement on a heated surface above the Leidenfrost regime. Int J Heat Mass Transf 104:1008-1021

Lakowicz JR (2013) Principles of fluorescence spectroscopy. Springer science $\&$ business media

Lemaitre P, Porcheron E, Grehan G, Bouilloux L (2006) Development of a global rainbow refractometry technique to measure the temperature of spray droplets in a large containment vessel. Meas Sci Technol 17:1299-1306

Lemoine F, Castanet G (2013) Temperature and chemical composition of droplets by optical measurement techniques: a state-of-the-art review. Exp Fluids 54:1572

Magde D, Rojas GE, Seybold PG (1999) Solvent dependence of the fluorescence lifetimes of xanthene dyes. Photochem Photobiol. https://doi.org/10.1111/j.1751-1097.1999.tb08277.x

Mendels DA, Graham EM, Magennis SW et al (2008) Quantitative comparison of thermal and solutal transport in a T-mixer by FLIM and CFD. Microfluid Nanofluidics. https://doi.org/10.1007/ s10404-008-0269-5

Mercadé-Prieto R, Rodriguez-Rivera L, Chen XD (2017) Fluorescence lifetime of Rhodamine B in aqueous solutions of polysaccharides and proteins as a function of viscosity and temperature. Photochem Photobiol Sci. https://doi.org/10.1039/c7pp00330g

Mishra YN, Abou Nada F, Polster S et al (2016) Thermometry in aqueous solutions and sprays using two-color LIF and structured illumination. Opt Express 24:4949

Müller T, Grünefeld G, Beushausen V (2000) High-precision measurement of the temperature of methanol and ethanol droplets using spontaneous Raman scattering. Appl Phys B 70:155-158

Müller CB, Weiß K, Loman A et al (2009) Remote temperature measurements in femto-liter volumes using dual-focus-Fluorescence Correlation Spectroscopy. Lab Chip. https://doi.org/10.1039/ b807910b

Natrajan VK, Christensen KT (2008) Two-color laser-induced fluorescent thermometry for microfluidic systems. Meas Sci Technol 20:15401 
Palmer J, Schumacher L, Reddemann MA et al (2020) Applicability of pulsed 2cLIF-EET for micro-droplet internal thermometry under evaporation conditions. Exp Fluids 61:1-18

Paviolo C, Clayton AHA, Mcarthur SL, Stoddart PR (2013) Temperature measurement in the microscopic regime: a comparison between fluorescence lifetime- and intensity-based methods. J Microsc. https://doi.org/10.1111/jmi.12033

Perrin L, Castanet G, Lemoine F (2015) Characterization of the evaporation of interacting droplets using combined optical techniques. Exp Fluids. https://doi.org/10.1007/s00348-015-1900-3

Rimbert N, Castanet G. Liquid Atomization out of a Full Cone Pressure Swirl Nozzle. 7th International Conference of Multiphase Flow (ICMF), Tampa, USA, 30 May to June 4, 2010.

Ross D, Gaitan M, Locascio LE (2001) Temperature measurement in microfluidic systems using a temperature-dependent fluorescent dye. Anal Chem 73:4117-4123

Saengkaew S, Godard G, Grehan G. Global Rainbow Technique : Temperature evolution measurements of super-cold droplets. 14th International Conference on Liquid Atomization and Spray Systems (ICLASS), Chicago, USA, 22-26 July, 2018.

Schweiger G (1990) Raman scattering on single aerosol particles and on flowing aerosols: a review. J Aerosol Sci 21:483-509

Smart PL, Laidlaw IMS (1977) An evaluation of some fluorescent dyes for water tracing. Water Resour Res 13:15-33

Stiti M, Labergue A, Lemoine F et al (2019) Temperature measurement and state determination of supercooled droplets using laserinduced fluorescence. Exp Fluids 60:1-13
Strizhak PA, Volkov RS, Antonov DV et al (2020) Application of the laser induced phosphorescence method to the analysis of temperature distribution in heated and evaporating droplets. Int $\mathrm{J}$ Heat Mass Transf. https://doi.org/10.1016/j.ijheatmasstransfer.2020. 120421

Stull R (2011) Wet-bulb temperature from relative humidity and air temperature. J Appl Meteorol Climatol 50:2267-2269

Tuckermann R, Bauerecker S, Cammenga HK (2005) IR-thermography of evaporating acoustically levitated drops. Int J Thermophys 26:1583-1594

Wolff M, Delconte A, Schmidt F et al (2007) High-pressure Diesel spray temperature measurements using two-colour laser-induced fluorescence. Meas Sci Technol. https://doi.org/10.1088/09570233/18/3/020

Zeng Y, Jiang L, Zheng W et al (2011) Quantitative imaging of mixing dynamics in microfluidic droplets using two-photon fluorescence lifetime imaging. Opt Lett. https://doi.org/10.1364/ol.36.002236

Zhang XF, Zhang Y, Liu L (2014) Fluorescence lifetimes and quantum yields of ten rhodamine derivatives: Structural effect on emission mechanism in different solvents. J Lumin 145:448-453

Publisher's Note Springer Nature remains neutral with regard to jurisdictional claims in published maps and institutional affiliations. 